\title{
Transcriptional activation of DBP by hnRNP K facilitates
}

\section{circadian rhythm.}

\author{
Paul Kwangho Kwon ${ }^{1}$, Kyung-Ha Lee ${ }^{2}$, Ji-hyung Kim ${ }^{3}$, Sookil Tae ${ }^{4}$, Seokjin Ham ${ }^{1}$, Hyo-Min Kim ${ }^{4}$, \\ Jung-Hyun Choi ${ }^{4}$, Young-Hun Jeong ${ }^{1}$, Sung Wook Kim ${ }^{4}$, Hee $\mathrm{Yi}^{5}$, Hyun-Ok Ku${ }^{5}$, Tae-Young Roh ${ }^{1,4}$, \\ Chunghun $\mathrm{Lim}^{3}$ and Kyong-Tai Kim ${ }^{1,4}$
}

${ }^{1}$ Department of Life Sciences, Pohang University of Science and Technology, Pohang, Gyeongbuk 37673, Republic of Korea.

${ }^{2}$ Division of Bio-technology and Convergence, Daegu Haany University (DHU), Gyeongsan-si, Gyeongbuk, Republic of Korea.

${ }^{3}$ School of Life Sciences, Ulsan National Institute of Science and Technology, 44919 Ulsan, Republic of Korea

${ }^{4}$ Division of Integrative Biosciences and Biotechnology, Pohang University of Science and Technology, Pohang, Gyeongbuk 37673, Republic of Korea.

${ }^{5}$ Vet Drugs and Biologics Division, Animal and Plant Quarantine Agency, 177, Hyeoksin 8-ro, Gimcheon-si, Gyeongsangbuk-do 39660, Republic of Korea

* To whom correspondence should be addressed. Tel: +82 54279 2297; Fax: +82 54279 2199; Email:

ktk@postech.ac.kr

\section{Abstract}

D-site albumin promoter binding protein (DBP) supports the rhythmic transcription of downstream genes, in part by displaying high-amplitude cycling of its own transcripts compared to other circadian clock genes. However, the underlying mechanism remains elusive. Here, we demonstrated that poly $(\mathrm{C})$ motif within DBP proximal promoters, in addition to an E-box element, provoked the transcriptional activation through increased RNA polymerase 2 (Pol2) recruitment by inducing higher chromatin accessibility. We also clarified that heterogeneous nuclear ribonucleoprotein $\mathrm{K}$ (hnRNP K) is a key regulator that binds to the poly $(C)$ motif on single-stranded DNAs in vitro. Chromatin immunoprecipitation further confirmed the expression-dependent and rhythmic binding of hnRNP $\mathrm{K}$ which was inhibited through its cytosolic localization mediated by time-dependent ERK activation. Knockdown of hnRNP K triggered low-amplitude mRNA rhythms in DBP and other core clock genes through transcriptional or post-transcriptional regulation. Finally, transgenic depletion of a Drosophila homolog of hnRNP K in circadian pacemaker neurons lengthened 24-hour periodicity in free-running locomotor behaviors. Taken together, our results provide new insights into the function of hnRNP $K$ as a transcriptional amplifier of DBP, which acts rhythmically through its intracellular localization by the 
ERK phosphorylation and as an mRNA stabilizer along with its physiological significance in circadian rhythms of Drosophila.

\section{Significance Statement}

In the case of mood disorders and the aging process, the mRNA expression and amplitude level of clock genes, including DBP, were reported to be diminished. However, the reason behind this decrease of clock gene amplitude and expression level remained unclear. Through this study, we revealed the regulatory mechanism behind the expression of clock genes, especially of DBP mRNA expression. In addition, we discovered that hnRNP K regulates more core clock genes than what we have previously known, such as Clock and Periods. Finally, we demonstrated the physiological significance of hnRNP $\mathrm{K}$ in Drosophila through its RNAi line model. Hence, our findings show the regulatory mechanism of circadian rhythm that may provide insight on mood disorder and aging process.

\section{Introduction}

Circadian rhythm is present in living organisms and reflects the elaborate nature of biological systems. Most living organisms have a circadian rhythm, which helps to synchronize daily changes in gene expression, metabolism, and physiology (1). The circadian clock system is modulated by transcriptional, post-transcriptional, translational, and post-translational regulation (2-4). Several studies have reported that clock genes are controlled by core genes such as the BMAL1-CLOCK complex, which acts as an activator $(5,6)$, and Cryptochrome (Cry1 and Cry2) $(7,8)$ along with Period (Per1, Per2, and Per3) (9-12) genes, which function as repressors. Recently, post-transcriptional regulation of clock genes by RNA-binding proteins such as hnRNP Q (13-15), PTB (16), hnRNP D (17), hnRNP A1 (18), hnRNP R (19), and hnRNP K (20) was shown to play a role in circadian rhythm.

Disruption of the amplitude of clock gene expression levels has been reported in studies of mood disorders and aging (21-24). Especially, both of $D$ site albumin promoter binding protein (DBP) oscillation amplitude and its expression level were decreased in fibroblasts from bipolar patients (21). Here, DBP mRNA oscillation is known for its high amplitude among clock genes $(25,26)$. DBP protein has a DNA binding domain that binds specifically to the D-box (TTATG[T/C]AA) and acts as a transcription activator (27-30). In mammalian genomes, there are more than 2,000 putative D-boxes, which govern about $10 \%$ of clock-controlled genes $(31,32)$. The oscillation of DBP mRNA is regulated by the BMAL1-CLOCK complex via multiple E-boxes (4, 33-35). Cry1 also affects the phase of DBP mRNA circadian rhythm (35). However, little is known about the mechanism of high-amplitude DBP mRNA oscillation.

As a strong poly $(\mathrm{C})$-binding protein (36), heterogeneous nuclear ribonucleoprotein $\mathrm{K}$ (hnRNP K) has been revealed to be involved in multiple gene expression processes when bound to singlestranded DNA or RNA, including the regulation of chromatin modification, transcription, post- 
transcriptional regulation, and translation (37). Among these multiple mechanisms of gene expression regulation, hnRNP K modulates transcription by interacting with TBP (TATA-binding protein) $(38,39)$ and by stabilizing DNA secondary structures as a trans-activator (40-42).

In this study, we investigated the reason behind high-amplitude DBP mRNA oscillation and the function of hnRNP $\mathrm{K}$ to support circadian rhythm in terms of transcriptional regulation. We further validated that the clock function of hnRNP $\mathrm{K}$ is conserved in circadian pacemaker neurons of Drosophila, where it sustains circadian locomotor behaviors.

\section{RESULTS}

\section{Oscillation of DBP mRNA expression is supported by its transcription at specific promoter regions.}

While the high amplitude of DBP mRNA oscillation has been reported, the reason behind the higher amplitude expression of DBP than that of other clock genes remained unclear $(25,26)$. First, we confirmed the oscillation of clock genes by treating NIH3T3 cells with dexamethasone synchronization for $36 \mathrm{hr}$ and measured the expression level of DBP pre-mRNA and mature mRNA (Figure S1A). In accordance with previous reports, we confirmed that DBP mature mRNA showed a slightly delayed peak time when compared to that of DBP pre-mRNA (43). Next, we measured the oscillation mRNA level of DBP and compared it to that of Rev-erba and Per2, which are known to be governed by E-box elements. We also compared the oscillation mRNA level of DBP to that of Bmal1, which has an antiphasic rhythm of expression and recognize E-box controlled genes (Figure S1B).

We hypothesized that the major factor that determines the high-amplitude of DBP mRNA expression is transcription efficiency via elevated transcriptional activation. To elucidate potential transcriptional regulatory motifs in eutherian mammals, we aligned the proximal promoter region (genomic regions of $1500 \mathrm{bp}$ from the transcription start site, TSS) of the DBP gene from multiple mammalian species using sequence data in the Ensembl database. We found that short promoter regions (-564 bp from the TSS) were highly alignable among eutherian mammals, suggesting that a possible transcriptional regulatory motif could exist in the proximal promoter region (Figure S1C). We cloned several deletion forms of the proximal promoter region for promoter assays with a luciferase reporter to determine regions critical for transcriptional activation at a peak time of DBP pre-mRNA. Our data indicated that P3 (-400), P4 (-450), P6 (which had lost 209 bp from the TSS, $\Delta-209$ ), wild-type (-564), and wild-type (Long) (-1500) promoter regions drove significant expression of luciferase, allowing us to determine specific regions of the proximal promoter critical for promoter activity (Figure 1A). In particular, P5 (which we deleted from 209 bp to 450 bp away from TSS, $\Delta-450 /-209)$ and P2 (-300) constructs had decreased promoter activity relative to that of P3 (-400), P4 (-450), P6 ( $\Delta-209)$, and wild-type (-564). Next, we identified the oscillation levels of $\mathrm{P} 2, \mathrm{P} 3$, and wild-type using real-time luminescence (Figure 1B). Our data showed that the region between P2 and P3 were necessary for high-amplitude oscillation of DBP transcription. This suggests that other crucial regions for efficient promoter activity exist in the region between $300 \mathrm{bp}$ and $400 \mathrm{bp}$ from the TSS apart from an E-box element. Therefore, 
we concluded that this specific region of the proximal promoter of DBP could possess critical regions for DBP transcriptional activation.

To identify transcriptional regulatory motifs, we used the MEME suite, a tool for searching for specific motifs. We scanned proximal promoter regions with MEME to reveal motifs between P2 (-300) and P3 $(-400)$ (Figure S2A). The poly (C) motif (or CCT motif) (Figure $1 \mathrm{C}$ ) was the only motif in this region predicted to function in transcription according to the GOMo tool (Figure 1D and Figure S2B).

Furthermore, high-amplitude oscillation of DBP mRNA is often found in mammals such as humans, mice, and rats $(21,27,44)$. Therefore, we aligned the poly $(C)$ motif sequences of several eutherian mammals, which revealed that the general transcriptional regulatory mechanism could be mediated by the poly $(C)$ motif (Figure 1E). Next, to identify levels of promoter activity, we cloned a mutated poly $(\mathrm{C})$ motif produced by random substitution from wild-type 5'CCGCCTCCAGCGCCTCCTCC3' to mutant 5'AGGAATATAGCGATTCATAA3' (Figure 1F). We measured the activity of wild-type and poly $(C)$ mutant promoters and found that DBP promoter activity of poly $(C)$ mutant was significantly decreased, which implied that poly $(C)$ motif played a key role in its promoter activity (Figure $1 G$ ). Next, we triggered oscillation by synchronizing cells with dexamethasone to identify differences between wild-type and poly $(\mathrm{C})$ mutant promoter activity using real-time luminescence. We found that the amplitude of luciferase expression by the poly $(C)$ mutant was markedly decreased compared to that of wild-type (Figure $1 \mathrm{H}$ ).

\section{The poly(C) motif is critical for high-amplitude DBP mRNA oscillation.}

Next, we designed an NIH3T3 cell line with a deletion of poly $(\mathrm{C})$ motif regions within DBP promoter using the CRISPR-Cas9 system to elucidate the endogenous function of these genomic regions during DBP mRNA oscillation (Figure 2A). After single cell sorting by GFP selection, poly(C) motif regions deleted cells were expanded and confirmed by sequencing in both forward and reverse directions (Figure 2B). We confirmed that the DBP mRNA oscillation amplitude of the poly(C) deleted cells was decreased compared to that of wild-type cells (Figure $2 \mathrm{C}$ ). We also confirmed that the amplitude of Per 2, which was known to be governed by the D-box element in addition to the E-box element (45), was decreased (Figure 2D). In contrast, Cry1 mRNA oscillation of poly(C) deleted cells were affected slightly when compared to wild-type cells (Figure 2E). Also, Clock mRNA oscillation level was not altered (Figure 2F).

To identify transcriptional regulation on DBP at the peak time, we measured the level of RNA Polymerase 2 (Pol2) binding during the peak time of DBP pre-mRNA oscillation. Control region of Pol2 ChIP was previously reported (46). The data showed that Pol2 binding level on the DBP promoter region was decreased in poly $(\mathrm{C})$ deleted cells (Figure $2 \mathrm{G}$ ).

Finally, to clarify the reason behind the reduced pol2 binding level on DBP promoter of poly $(C)$ deleted cells, we utilized the Assay for Transposase-Accessible Chromatin (ATAC) along with realtime PCR. Interestingly, ATAC-qPCR data showed that chromatin accessibility levels were regulated by poly $(C)$ motif region depending on the circadian rhythm (Figure $2 \mathrm{H}$ ). These results propose that DBP mRNA oscillation might be facilitated by the modulation of chromatin accessibility mediated by poly $(\mathrm{C})$ motif regions. 


\section{hnRNP K binds to the single-stranded poly(C) motif directly and rhythmically.}

To demonstrate the poly $(\mathrm{C})$ motif binding factor, we pulled down proteins that were bound onto poly $(C)$ oligonucleotide. Descriptions of the forward and reverse poly $(C)$ motif sequences are provided in Figure 3A. We attached biotin to the 5' nucleotide (Solgent, South Korea) of the forward sequence 5'CCGCCTCCAGCGCCTCCTCC3' and the reverse complementary sequence 5'GGAGGAGGCGCTGGAGGCGG3' and performed pull-down experiments with streptavidin in HEK293A cells. We found that almost no proteins were pulled down with the duplex form of the oligonucleotide (data not shown). Thus, we presumed that the single strand oligonucleotide of the poly $(C)$ motif might interact with certain unknown transcription factors. Surprisingly, we identified that a single, thick protein band was detected when we used the forward poly $(\mathrm{C})$ motif single-strand oligonucleotide, which was identified as hnRNP K by mass spectrometry (Figure 3A, Figure S3A). The CCT element is known to be a target of hnRNP K, a single-stranded DNA binding protein that functions as a regulator of the expression of multiple genes $(37,38,40,41)$. We also detected the binding of hnRNP $\mathrm{K}$ using anti-hnRNP $\mathrm{K}$ at the poly(C) motif (Figure S3B).

To confirm direct binding of hnRNP $K$ to the poly $(C)$ motif oligonucleotide, we purified GST and GSThnRNP $\mathrm{K}$ proteins and performed pull-down experiments with 5' biotinylated poly $(\mathrm{C})$ motif and 5' biotinylated poly $(\mathrm{C})$ mutant (Figure 3B). We confirmed that GST-hnRNP K was detected only in the wild-type oligonucleotide pulled-down group through immunoblotting (Figure 3B). This demonstrated that hnRNP $\mathrm{K}$ binds directly to the poly $(\mathrm{C})$ motif of DBP promoter regions in vitro.

Although the CCT element has already been reported to be a binding target of hnRNP K, functional confirmation of this interaction and prediction of the function of hnRNP $\mathrm{K}$ on this motif has not previously been investigated $(37,38,40,41)$. For this reason, we searched a database that contains 1056 hnRNP K-bound promoter genes from hnRNP K ChIP-Seq (47) for specific motifs that were predicted to function in transcription using MEME-ChIP (Figure S3C). We detected poly(C) and CCT motifs within the proximal promoter regions of hnRNP K-bound promoter genes (Figure 3C), both of which are predicted to function as transcription (Figure S3C). This suggests that hnRNP K binds to single-stranded poly $(\mathrm{C})$ and CCT motifs and may, therefore, function as a transcription activator.

Next, we analyzed the KH3 domain sequence of hnRNP K (Figure S4A), which is a single-stranded DNA binding domain (48-50). Using the Ensembl database, we found that the $\mathrm{KH} 3$ domain of several eutherian mammals aligned perfectly (Figure S4B). This result suggests that hnRNP K binding to the promoter region of DBP could be a critical regulatory mechanism for DBP transcription in eutherian mammals.

Previously, we confirmed that hnRNP $\mathrm{K}$ bound to the poly $(\mathrm{C})$ motif in vitro and predicted that this would allow hnRNP $\mathrm{K}$ to function as a transcriptional activator using Gene Ontology for Motifs (GOMo). We then examined the endogenous binding of hnRNP $\mathrm{K}$ to the proximal promoter region of DBP using a Chromatin Immunoprecipitation (ChIP) assay, because previously reported ChIP-seq data did not show DBP as a target gene of hnRNP K. The ChIP results showed that hnRNP K specifically bound to Region 2 (R2), which contains a poly(C) motif between -300 and -400 from the TSS. Region 1 (R1) and Region 3 (R3) were not bound by hnRNP K (Figure 3D). The RB1 gene, to 
which hnRNP K binds robustly, was used as a positive control, while Gapdh was used as a negative control (47). Additionally, we investigated whether the binding of hnRNP K was rhythmically influenced by dexamethasone synchronization, which induces mRNA circadian oscillation of clock genes, to further clarify the involvement of hnRNP $\mathrm{K}$ in DBP transcription. We found that oscillation of DBP pre-mRNA levels correlated with the rhythmic binding of hnRNP $\mathrm{K}$ to R2 (Figure 3E). Furthermore, we confirmed the subcellular protein expression levels of hnRNP $K$ by subcellular fractionation of NIH3T3 cells. These data suggest that the rhythmic expression of nuclear hnRNP $K$ proteins may be a major contributing factor to the rhythmic binding of hnRNP K (Figure 3F and 3G).

Because it was previously reported that hnRNP K transport was controlled by an ERK activation and that ERK activation was mediated in rhythmic manners, we demonstrated the time-dependent transport of hnRNP $\mathrm{K}$ along with activation level of ERK (51-53). Interestingly, we identified that the peak time of ERK activation level was inversely related with the peak time of hnRNP K nuclear expression level after dexamethasone synchronization (Figure $3 \mathrm{H}$ ). Next, we overexpressed hnRNP $\mathrm{K}$ WT and hnRNP $\mathrm{K}$ phosphomutant (which was a direct target site of phosphorylation by ERK activation) at both the peak time (Figure $3 \mathrm{H}$ ) and trough time (Figure S4C) of ERK phosphorylation. The result showed that phosphomutant hnRNP $K$ raised DBP mRNA levels at the peak time of ERK activation, but not the wildtype hnRNP $\mathrm{K}$, which meant that phosphorylation of hnRNP $\mathrm{K}$ by ERK activation inhibited transcription in the nucleus by transporting hnRNP $\mathrm{K}$ to the cytoplasm.

Thus, our data suggest that time-dependent ERK activation controls hnRNP K transport, which regulates the binding of hnRNP $K$ onto poly $(C)$ motif regions resulting in the robust oscillation of DBP mRNA. This finding led us to explore possible mechanisms of high-amplitude DBP mRNA expression.

\section{hnRNP K expression level controls the transcription of DBP through the poly(C) motif.}

We then assessed promoter activity through knockdown of hnRNP K. The knockdown efficiency of the hnRNP K siRNA pool was measured by immunoblotting (Figure 4A). Cells transfected with the hnRNP K siRNA pool showed an approximately $70 \%$ decrease in hnRNP K protein levels than the siRNA-transfected control group. Furthermore, we checked cell viability using the MTT assay. Under our experimental conditions, knockdown of hnRNP K did not significantly affect cell viability (Figure 4B).

We compared the levels of DBP-luciferase constructs containing all regulatory elements including the poly $(\mathrm{C})$ motif, along with wild-type and wild-type (Long) constructs, during knockdown of hnRNP K. We confirmed that levels of DBP-luciferase, wild-type, and wild-type (Long) expression were downregulated by knockdown of hnRNP K (Figure 4C). Next, we measured the promoter activity of constructs that contained serially deleted proximal promoter regions as shown in Figure $1 \mathrm{~A}$. We also confirmed that the promoter activities of the P3, P4, P6, and wild-type constructs decreased significantly when hnRNP K was downregulated (Figure 4D). A poly (C) motif was present in the P3, P4, P6, and wild-type promoters, which implies a correlation between the poly $(C)$ motif and hnRNP K. Next, we conducted a promoter assay with the wild-type and poly $(\mathrm{C})$ mutant shown in Figure $1 \mathrm{~F}$ during knockdown of hnRNP K. While this clearly decreased the promoter activity of the wild-type, the 
promoter activity of the poly $(\mathrm{C})$ mutant changed only a little (Figure $4 \mathrm{E})$. We also overexpressed hnRNP $K$ using a human influenza hemagglutinin (HA) tag to assess promoter activity. Overexpression of hnRNP K increased wild-type promoter activity only (Figure 4F). This indicates that hnRNP $\mathrm{K}$ could function as a transcriptional activator by binding to the poly $(\mathrm{C})$ motif in the DBP promoter.

\section{hnRNP K supports high-amplitude DBP oscillation by influencing transcription, not mRNA stability.}

After clarifying DBP promoter activity through luciferase reporter assays, we measured the endogenous levels of DBP pre-mRNA and mature mRNA. Levels of both these types of DBP mRNA decreased after knockdown of hnRNP K (Figure 5A). Per3, which has been reported to retain mRNA stability through hnRNP K, was used as a positive control (20). In addition, we elucidated the kinetics of mRNA decay to exclude the possibility of mRNA degradation, which was previously reported to be related to the function of hnRNP $K(20)$. To examine the role of hnRNP $K$ in DBP regulation, the mRNA stability of the control group and the siRNA knockdown group were compared under treatment with actinomycin $D$, which inhibits transcription. The half-life of DBP mRNA did not change during the knockdown of hnRNP $\mathrm{K}$ (Figure $5 \mathrm{~B}$ ). This indicates that hnRNP $\mathrm{K}$ regulates DBP not by mRNA degradation, but by transcriptional activation.

The circadian rhythm of DBP mRNA in mouse NIH3T3 cells was also measured after triggering oscillation with dexamethasone to clarify the role of hnRNP $\mathrm{K}$ in maintaining high-amplitude DBP mRNA oscillation. Quantification of circadian rhythm oscillation under hnRNP K knockdown revealed a decrease in DBP mRNA oscillation amplitude (Figure $5 \mathrm{C}$ ). This result indicates that high-amplitude DBP oscillations are maintained through transcriptional activation by hnRNP K.

Next, we examined the effects of hnRNP K on other D-box trans-acting regulators-thyrotroph embryonic factor (Tef), hepatic leukemia factor (Hlf), and nuclear factor interleukin 3 regulated (Nfil3) - by measuring mRNA levels during knockdown of hnRNP K. We confirmed that the mRNA level of DBP decreased significantly during knockdown of hnRNP $K$, while mRNA levels of Tef, Hif, and Nfil3 did not (Figure S5A). This indicates that the primary target of hnRNP $K$ is the D-box regulator $\mathrm{DBP}$, not other $\mathrm{D}$-box regulators.

Previously, we revealed that poly $(\mathrm{C})$ motif regions among eutherian mammals could function as the binding site of transcriptional activators. We found that the mRNA level of hnRNP $\mathrm{K}$ in human U2OS cells was $30 \%$ of that of the control when hnRNP $\mathrm{K}$ was knocked down (Figure S5B). Furthermore, cell viability testing by the MTT assay demonstrated that knockdown of hnRNP K did not significantly affect cell viability under our experimental conditions (Figure S5C). Oscillation amplitude of human DBP mRNA was also decreased during hnRNP K knockdown (Figure 5D).

Finally, we investigated the mRNA levels of other core clock genes, including Bmal1, Clock, Cry1, Cry2, Period1, and Period2 during the knockdown of hnRNP K. Interestingly, even though the mRNA level of Bmal1, Cry1, and Cry2 were not changed significantly, mRNA levels of Clock, Period1 and Period 2 were down-regulated during knockdown of hnRNP K (Figure S5D). Next, we confirmed that 
mRNA oscillation levels of Per1, Per2, and, Clock. We found that mRNA oscillation levels of Per1, Per2, and Clock were lowered after Dexamethasone synchronization during knockdown of hnRNP $\mathrm{K}$ (Figure 5E-5G). In contrast, Cry1 mRNA oscillation level was only slightly changed (Figure S5E). Also, Bmal1 mRNA was only marginally lowered during the knockdown of hnRNP K (Figure S5F). Interestingly, we measured mRNA decay kinetics of other clock genes, which demonstrated that Clock might be controlled post-transcriptionally by hnRNP K, while Per1 and Per2 showed marginal difference (Figure 5H-5J).

In conclusion, we propose that DBP mRNA oscillation, which is mediated by hnRNP $\mathrm{K}$, also affects other core clock genes such as Clock and Periods, transcriptionally and post-transcriptionally. This leads us to assume that the rhythmic behaviors of an organism could be affected during the knockdown of hnRNP K.

\section{hnRNP K depletion in Drosophila circadian pacemaker neurons impairs circadian behaviors.}

hnRNP K homologs are well conserved in other species including Drosophila (37). Moreover, it has been demonstrated that Drosophila hnRNP K proteins associate selectively with poly $(\mathrm{C})$ nucleotides $(54,55)$. We, therefore, took a genetic approach in Drosophila to determine if hnRNP K-dependent clock function is conserved in non-mammalian species and sustains circadian rhythm at the organismal level. To this end, we quantitatively analyzed circadian locomotor activities in individual flies as a readout of the robustness of circadian rhythms.

Since a strong hypomorphic mutation in the Drosophila hnRNP K gene causes lethality $(55,56)$, we expressed an RNA interference transgene against hnRNP $\mathrm{K}$ in different groups of circadian pacemaker neurons and examined its effects on circadian locomotor rhythms in adult flies. We first found that hnRNP $\mathrm{K}$ depletion in a small number of circadian pacemaker neurons called posterior dorsal neurons 1 (DN1p) (57-60) (by R18H11-Gal4) suppressed evening activity peak anticipatory to lights-off in 12-hour light: 12-hour dark (LD) cycles (Figure 6A, top). Transgenic flies were further transferred from LD cycles to constant dark (DD) where their rhythmic locomotor activities were primarily driven by endogenous circadian clocks. In the first DD cycle, the hnRNP $\mathrm{K}$ depletion evidently delayed the circadian phases of morning and evening activity peaks (Figure 6A, middle), leading to long-period rhythms in the subsequent DD cycles (Figure $6 \mathrm{~A}$, bottom). Behavioral analyses using three independent RNAi transgenes consistently validated that hnRNP $K$ depletion in DN1p neurons lengthened the circadian periodicity in DD locomotor rhythms by $\sim 2$ hours (Figure $6 B$ ) while weaker rhythmicity was observed only in one of the three RNAi lines (Figure 6C).

By contrast, we could not observe any comparable phenotypes in circadian behaviors when hnRNP $\mathrm{K}$ was depleted in either circadian pacemaker cells broadly expressing TIMELESS (by tim-Gal4) (61) or lateral neurons expressing a circadian neuropeptide PIGMENT-DISPERSING FACTOR (by PdfGal4) (62) (Figure S6A and S6B). We reason that possible differences in relative abundance of hnRNP K expression and/or efficiency of its transgenic depletion among different groups of circadian pacemaker neurons might explain the long-period rhythms caused selectively by the strong DN1p Gal4 driver. Alternatively, but not exclusively, DN1p neurons might specifically express a clock- 
relevant gene of which the expression is regulated by hnRNP $\mathrm{K}$ and thereby limiting the 24-hour periodicity in DD locomotor rhythms of hnRNP K-depleted flies. Nonetheless, these data demonstrate that hnRNP $\mathrm{K}$ homologs sustain circadian rhythms in both flies and mammals, although genetic or neural substrates of hnRNP K-dependent clocks might be diverse.

\section{DISCUSSION}

One of the main findings in this study is that high-amplitude DBP mRNA oscillations are mediated by hnRNP K, which functions as a transcriptional activator. Prior to this study, it was reported that the Bmal1-Clock complex is the main regulator of rhythmic DBP mRNA expression via multiple E-boxes (4, 33-35). Here, we unveiled an additional and novel regulating mechanism that maintains highamplitude oscillation. A recent study measured rhythmic H3K27ac enrichment and Pol2 density within the DBP proximal promoter regions using ChIP-seq (63). Together with our study findings, this suggests that DBP rhythmic transcription is regulated through a combined regulatory mechanism involving hnRNP $\mathrm{K}$. We demonstrated that hnRNP $\mathrm{K}$ could indeed affect the circadian rhythm oscillation of DBP mRNA by acting as a transcription activator along with other complex regulating mechanisms.

Although the importance of maintaining the amplitude of clock gene oscillations has been established, the mechanisms underlying this have remained unclear. Therefore, we investigated the oscillation pattern of DBP and compared it to that of other clock genes (Figure S1A and S1B). Next, we aligned the genomic region upstream of the TSS of DBP among eutherian mammals to determine transcriptional regulatory motifs (Figure S1C). Analysis of the promoter alignment using the MEME suite revealed a poly $(\mathrm{C})$ motif that was predicted to be a transcriptional activator. We confirmed the promoter activity and function of the poly $(\mathrm{C})$ motif using luciferase reporter assays and the CRISPRCas9 system (Figure 2). At the same time, we confirmed the presence of the poly $(C)$ motif in proximal promoter regions by analyzing hnRNP K ChIP-ChIP data using MEME-ChIP $(47,64)$ (Figure 3C). This novel approach towards motif analysis provides a systematic way to confirm predictions experimentally.

hnRNP $\mathrm{K}$ binds to single-stranded DNA to stabilize the secondary structure of DNA, thereby promoting transcription (42). Here, we determined hnRNP $\mathrm{K}$, known to be present in yeast to mammals $(36,37)$, to be the binding partner of the poly $(C)$ motif using an oligonucleotide pulldown assay (Figure $3 \mathrm{~A}$ and $3 \mathrm{~B}$ ). We also confirmed the binding of endogenous hnRNP $\mathrm{K}$ to the poly $(\mathrm{C})$ motif in the DBP proximal promoter region, as well as its rhythmic interaction during circadian oscillation (Figure 3D and $3 \mathrm{E}$ ). This suggests that the poly $(\mathrm{C})$ motif and hnRNP K might function as critical regulators of circadian rhythm in eutherian mammals. Moreover, we found that the regulation of DBP promoter activity was dependent on the level of nuclear hnRNP K (Figure 4). Also, hnRNP K transport level by ERK activation, in addition to its protein expression levels, could affect its rhythmic binding to the poly $(\mathrm{C})$ motif, resulting in high-amplitude DBP oscillation (Figure $3 \mathrm{H}$ ).

Furthermore, we measured the endogenous DBP mRNA level after knockdown of hnRNP $\mathrm{K}$ (Figure 5A). We demonstrated a decrease in the amplitude of DBP mRNA oscillation by suppressing 
the level of hnRNP $\mathrm{K}$ in mouse and human cells (Figure 5C and 5D). We, therefore, suggest that circadian rhythm is maintained by the level of hnRNP K. In summary, we demonstrated hnRNP Kmediated transcriptional regulation of DBP, which is critical for the robust maintenance of high amplitude DBP mRNA oscillations.

This study provides the first evidence that the relationship between hnRNP $\mathrm{K}$ and DBP is crucial for transcription and circadian rhythm. In addition to this, we verified mRNA oscillations of other core clock genes that are controlled by post-transcriptional or transcriptional regulation during low-level of hnRNP K (Figure 5H-5J). Here, we investigated the mechanistic relationship between hnRNP K and DBP that results in delicate control of circadian rhythm in complicated biological systems. Although the precise mechanisms of rhythmic interaction and the involvement of hnRNP $\mathrm{K}$ in rhythmic expression remain to be identified, we showed that hnRNP K-mediated transcription and posttranscriptional regulation impacts the oscillation of clock genes and proposed conservation of hnRNP K-dependent clocks.

\section{MATERIAL AND METHODS}

\section{Cell culture and drug treatment}

NIH 3T3 cells, HEK293A, and U2OS cells were cultured in Dulbecco's modified Eagle's medium (HyClone) supplemented with 10\% fetal bovine serum (HyClone) and $1 \%$ antibiotics (WelGENE) and were maintained in a humidified incubator with 95\% air and 5\% CO2. NIH 3T3 and U2OS cells were synchronized by treatment with $100 \mathrm{nM}$ dexamethasone in 12 -well plates containing $\sim 3.0 \times 10^{5}$ cells per well. After $2 \mathrm{hrs}$, the medium was replaced with complete medium and the cells were harvested every 6 hrs for each sample (15). To measure mRNA decay kinetics, we blocked transcription by treating actinomycin D $(5 \mu \mathrm{g} / \mathrm{ml})$ to the $\mathrm{NIH} 3 \mathrm{~T} 3$ cells and harvested them every $2 \mathrm{hrs}$ for each sample.

\section{Plasmids}

To generate the pGL3 vector which contains promoters of DBP, we amplified the several forms of mouse Dbp promoters (UCSC Genome Browser on Mouse Dec. 2011 (GRCm38/mm10) Assembly, https://genome.ucsc.edu) with forward and reverse primers using Pfu polymerase (Solgent, Daejeon, South Korea). The PCR products were digested with Nhe1 and Hind3 and then inserted into the pGL3 basic vector, yielding pGL3 with the short form of Dbp promoter and pGL3 with the long form of Dbp promoter, which was confirmed by sequencing. Serial deletion constructs (P1, P2, P3, P4, P5, P6) and mutant constructs were cloned in the same way. Dbp-luciferase construct was kindly gifted by Ripperger (Stratmann et al., 2010). HA-hnRNP K and HA-hnRNP K (S284A/S353A) were kindly provided by Ronai (Habelhah et al., 2001).

\section{RNA extraction \& quantitative PCR}

Total RNA was extracted from NIH 3T3 and U2OS cells using the TRI Reagent (Molecular Research Center, Bio Science Technology). Next, we treated RQ1 RNase-free DNase (Promega) after RNA 
quantification to remove contaminated DNA according to the manufacturer's instructions. RNA $(1 \mu \mathrm{g})$ was reverse-transcribed using ImProm-II ${ }^{\mathrm{TM}}$ (Promega) according to the manufacturer's instructions. Endogenous mRNA levels were detected by quantitative real-time PCR using the StepOnePlus realtime PCR system (Applied Biosystems) with FastStart Universal SYBR Green Master (Roche), as described previously (65). Specific primer pairs for mouse Dbp (NM_016974.3), mouse pre-Dbp which primers targeted for intron regions (4), mouse Per1 (NM_011065.5 mouse Per2 (NM_011066.3), mouse Rev-erba (NM_145434.4), mouse Hlf (NM_172563.3), mouse Tef (NM_017376.3), mouse Nfil3 (NM_017373.3) (18), mouse Bmal1 (NM_007489.4), mouse Per3 (NM_001289877.1) (20), mouse Cry1 (NM_007771.3), mouse Cry2 (NM_009963.4), human hnRNP K (NM_002140.4), and human DBP (NM_001352.4) were used for real-time PCR (the primer sequences are shown in Supplementary Table S1).

\section{Luciferase assay}

Firefly and Renila luciferase activities were determined by using the Dual-Luciferase(®) Reporter Assay System created by Promega (Promega Corporation, Wisconsin, USA) according to the manufacturer's instructions. Normalized FLUC activity was determined as the ratio of Firefly to Renila activity.

\section{Real-time luminescence}

$5 \times 10^{5} \mathrm{NIH} 3$ T3 fibroblasts (ATCC) were plated in $35 \mathrm{~mm}$ dishes. Cells were transfected with $1 \mu \mathrm{g}$ of each mDBP-luciferase construct using Lipofectamine 2000 (Invitrogen) according to the manufacturer's instructions. After overnight culture, $100 \mathrm{nM}$ dexamethasone was added to the cells and cells were incubated for $2 \mathrm{hr}$. The medium was then changed to DMEM without phenol red and 1 $\mathrm{mM}$ luciferin (Promega). A Lumicycle device (Actimetrics) kept in the $37^{\circ} \mathrm{C}$ incubator for recording measured luminescence for 3 or 4 days. Lumicycle analysis software (Actimetrics) was used for lumicycle data analysis.

\section{Generation of poly(C) motif region-deleted cell lines}

We used the pSpCas9(BB)-2A-GFP (PX458) plasmid obtained from Addgene (Addgene, \#48138) for Cas9-CRISPR experiments. We designed sgRNA sequences to delete poly(C) motif containing regions within the DBP promoter (66) by using the online CRISPR Design tool (http://crispr.mit.edu/). Sequences were as follows: Target 1 (Forward : 5'-CACCGCATTGGCCCGAAGTGGGTCA-3, Reverse : 5'-CTGACCCACTTCGGGCCAATGCAAA-3') and Target 2 (Forward : 5'CACCGGCGGGACACTGACCTATATT-3;, Reverse : 5'-CAATATAGGTCAGTGTCCCGCCAAA-3'). Then, genomic DNA sequencing was performed with the following primers: Forward, CTA GCT AGC CGA TAG CAC GCG CAA AGC CA; reverse, 5'-CCC AAG CTT GGC AAG AAC CAA TCA CGT CT3 ' to confirm genomic deletion.

\section{ChIP assay}


After fixation of $10^{7}$ cells of $\mathrm{NIH} 3 \mathrm{~T} 3$ cells by $1 \%$ formaldehyde at RT for $10 \mathrm{~min}, 0.125 \mathrm{M}$ glycine was treated for $10 \mathrm{~min}$ to quench the formaldehyde. Next, the fixed cells were suspended in $10 \mathrm{mM}$ Tris-Cl, pH 8.0, 1 mM EDTA, 0.1\% SDS, 0.5 mM PMSF, and protease inhibitor cocktail (Roche). Crosslinked cells were sonicated by a sonicator (VibraCell, Sonics, USA) with 20 cycles of sonication (30 sec) and resting $(30 \mathrm{sec})$ at an amplitude of 8 . Sonicated chromatin was analyzed by agarose gel electrophoresis to ensure that DNA fragment sizes did not exceed $500 \mathrm{bp}$. Anti-hnRNP K antibody (ab70492, Abcam) and Pol2 (8WG16, Abcam) was added to $750 \mathrm{ul}$ of chromatin and incubated at $4^{\circ} \mathrm{C}$ for 2 hrs with rotation. $30 \mu \mathrm{l}$ of Protein G magnetic beads (88848, Thermo) was added to the chromatin-antibody mixture and were incubated at $4^{\circ} \mathrm{C}$ overnight with rotation. Magnetic beads were washed twice with $1 \mathrm{ml}$ of ChIP wash buffer 1 (10 mM Tris-Cl, pH 7.4, $1 \mathrm{mM}$ EDTA, 0.1\% SDS, 0.1\% sodium deoxycholate, $1 \%$ Triton X-100) for $10 \mathrm{~min}$, twice with $1 \mathrm{ml}$ of ChIP wash buffer 2 (10 mM Tris$\mathrm{Cl}, \mathrm{pH} 7.4,1 \mathrm{mM}$ EDTA, $0.1 \%$ SDS, $0.1 \%$ sodium deoxycholate, $1 \%$ Triton $\mathrm{X}-100,0.3 \mathrm{M} \mathrm{NaCl}$ ) for 10 min, twice with $1 \mathrm{ml}$ of ChIP wash buffer $3(10 \mathrm{mM}$ Tris-Cl, pH 7.4, $1 \mathrm{mM}$ EDTA, 0.25M LiCl, 0.5\% NP$40,0.5 \%$ sodium deoxycholate) for $10 \mathrm{~min}$, once with $1 \mathrm{ml}$ of ChIP wash buffer 4 (10 mM Tris- $\mathrm{Cl}, \mathrm{pH}$ 7.4, $1 \mathrm{mM}$ EDTA, $0.2 \%$ Triton X-100) for $10 \mathrm{~min}$, and once with $1 \mathrm{ml}$ of $1 \mathrm{X}$ TE buffer (10 mM Tris-Cl, $\mathrm{pH} 7.4,1 \mathrm{mM}$ EDTA) for $10 \mathrm{~min}$. Washed beads were pelleted on a magnetic stand and the clear supernatant was carefully removed and discarded. Beads were resuspended in $200 \mu \mathrm{l}$ of ChIP elution buffer (50mM Tris-Cl, pH 7.5, $10 \mathrm{mM}$ EDTA, 1\% SDS) and 5 $\mu$ l of proteinase K (Roche, Cat \# 03115 828001 ) was added to beads suspension. The beads and proteinase $\mathrm{K}$ were mixed well by pipetting and were incubated at $65^{\circ} \mathrm{C}$ for $5 \mathrm{hrs}$. $200 \mu \mathrm{l}$ of phenol:chloroform: isoamyl alcohol (25: 24: 1) (Affymetrix, Cat. \# $75831400 \mathrm{ML}$ ) was added to beads suspension and mixed vigorously by vortexing for $30 \mathrm{sec}$ and was centrifuged at 13,000 rpm for $15 \mathrm{~min}$ under room temperature. After centrifugation, the upper aqueous phase was transferred to a fresh $1.5 \mathrm{ml}$ tube and $2 \mu \mathrm{l}$ of glycogen solution (Roche, Cat. \# 10901393001), 20 $\mathrm{\mu l}$ of $3 \mathrm{M}$ sodium acetate, and $500 \mu \mathrm{l}$ of $100 \%$ ethanol were added and the solution was incubated at $-20^{\circ} \mathrm{C}$ overnight. DNA was pelleted by centrifuging at $13,000 \mathrm{rpm}$ for $30 \mathrm{~min}$ at $4^{\circ} \mathrm{C}$. The supernatant was carefully removed and discarded. DNA pellet was washed with $700 \mu \mathrm{l}$ of $70 \%$ ethanol and DNA was pelleted by centrifuging at $13,000 \mathrm{rpm}$ for $15 \mathrm{~min}$ at $4^{\circ} \mathrm{C}$. The supernatant was carefully removed and discarded while the DNA pellet was air-dried for $15 \mathrm{~min}$ at room temperature. DNA was resuspended in $50 \mu$ l of $1 \mathrm{X}$ TE buffer (10mM Tris-Cl, pH 7.5, 1mM EDTA) and used for quantification by quantitative PCR (the primer sequences are shown in Supplemental Table S2).

\section{ATAC-qPCR}

ATAC-seq was performed as described (67) on four of wild-type NIH3T3 and four of poly(c) deleted NIH3T3. Libraries were generated using the Ad1_noMX, Ad2.13, Ad2.15, Ad2.16 and Ad2.17 barcoded primers from Buenrostro et al. (68) and were amplified for 6-7 total cycles. Libraries were purified with SPRI beads to remove remaining primer dimers. Libraries were quantitated by using Qubit. 


\section{Subcellular fractionation}

Hypotonic buffer (10mM HEPES (pH7.9), 10mM KCl, 1.5mM MgCl2, DTT 1.0mM, 0.2mM PMSF) was added to cells to resuspend them after cell harvest. Then, Lysis buffer (10mM HEPES (pH7.9), 10mM $\mathrm{KCl}, 1.5 \mathrm{mM} \mathrm{MgCl} 2,1.0 \mathrm{mM}$ DTT, 0.2mM PMSF, $2.5 \% \mathrm{NP}-40$ ) was added and the solution was centrifuged at $3500 \mathrm{rpm}, 4^{\circ} \mathrm{C}$ for $4 \mathrm{~min}$. Cytoplasmic fraction was extracted with 5 trials of freezing and thawing of the supernatant of cell lysate followed by centrifugation at $15000 \mathrm{rpm}, 4^{\circ} \mathrm{C}$, for $20 \mathrm{~min}$. Next, to extract nuclear fraction, extraction buffer (20mM HEPES $(\mathrm{pH} 7.9), 450 \mathrm{mM} \mathrm{NaCl}, 1.5 \mathrm{mM}$ MgCl2, 1.0mM DTT, 0.2mM PMSF, 0.2mM EDTA ( $\mathrm{mH} 8.0$ ) was added to pellet of cell lysate and were centrifuged at $15000 \mathrm{rpm}, 4^{\circ} \mathrm{C}$, for $20 \mathrm{~min}$ after 5 trials of freezing and thawing. Each fraction was quantified by Bradford assay and were boiled at $95^{\circ} \mathrm{C}$ for 5 min with sample buffer $(40 \%$ Glycerol, $240 \mathrm{mM}$ Tris/ $\mathrm{HCl} \mathrm{pH} \mathrm{6.8,8 \%} \mathrm{SDS,} \mathrm{0.04 \%} \mathrm{bromophenol} \mathrm{blue,} 5 \%$ beta-mercaptoethanol).

\section{Transient transfection and RNA interference}

The Neon® Transfection System (Invitrogen) was used for transient transfection as recommended by the manufacturer, and Metafectene (Bionex) was used according to the manufacturer's instruction. We transfected cells with $2 \mu \mathrm{g}$ of luciferase reporter plasmid and maintained the cells in 6-well plates. For hnRNP $\mathrm{K}$ knockdown or overexpression and subsequent luciferase assays, we transfected cells with $1 \mu \mathrm{l}$ of $20 \mu \mathrm{M}$ siRNA or $1 \mu \mathrm{g}$ of hnRNP K-overexpressing vectors (HA-pcDNA vectors) $12 \mathrm{hr}$ after transfection of cells with $1 \mu \mathrm{g}$ luciferase reporter plasmid. We also used specific small interfering RNAs (siRNAs) for hnRNP $\mathrm{K}$ siRNA pools (sihnRNP $\mathrm{K} 1$ sense : 5'aaaggaggcaagaauauua(dTdT)3', antisense : 5'uaauauucuugccuccuuu(dTdT)3', sihnRNP K2 sense : 5'gcuguggaaugcuuaaauu(dTdT)3', antisense : 5'aauuuaagcauuccacagc(dTdT)3', sihnRNP K3 sense : 5'cugaugagaugguugaauu(dTdT)3', antisense : 5'aauucaaccaucucaucag(dTdT)3', sihnRNP K4 sense : 5'ucaugaaucuggagcauca(dTdT)3', antisense : 5'ugaugcuccagauucauga(dTdT)3') (BIONEER, South Korea) to conduct knockdown of hnRNP K.

\section{Oligonucleotide pull-down assay and mass spectrometry analysis}

We used biotinylated oligonucleotides (Solgent, South Korea) for oligonucleotide pull-down assays. The biotinylated oligonucleotides had the following sequences: biotinylated wild-type, 5'-CCG CCT CCA GCG CCT CCT CC-3' and biotinylated mutant, 5'-AGG AAT ATA GCG ATT CAT AA-3'. After harvesting HEK293A cells, we lysed the cells using RIPA buffer (10 mM Tris-Cl, pH 7.4, 1 mM EDTA, $0.1 \%$ SDS, $0.1 \%$ sodium deoxycholate, $1 \%$ Triton $X-100$ ) and centrifuged them at 15,000 rpm for 15 $\min$ at $4^{\circ} \mathrm{C}$. The supernatant was used as the cell lysate for the binding assay. First, we washed streptavidin agarose beaded resin (Thermo Scientific, Cat \# 20349) and biotinylated oligonucleotides with RIPA buffer. Then we allowed binding of the streptavidin-agarose beaded resin to the biotinylated oligonucleotides and the cell lysate, GST, or GST-hnRNP K simultaneously for $1 \mathrm{hr}$ to increase the specificity of pulldown. Next, we mixed streptavidin agarose beaded resin bound by biotinylated 
oligonucleotide with either precleared cell lysate, GST, or GST-hnRNP K to allow binding at $4^{\circ} \mathrm{C}$ for 4 hrs. The resin was then washed three times with RIPA buffer and boiled in sample buffer $(40 \%$ glycerol, $240 \mathrm{mM}$ Tris/HCl pH 6.8, 8\% SDS, 0.04\% bromophenol blue, 5\% beta-mercaptoethanol) at $95^{\circ} \mathrm{C}$ for $10 \mathrm{~min}$. Finally, SDS-PAGE was conducted. After SDS-PAGE, the gel was stained with Brilliant Blue R-250 (Sigma-Aldrich) for $30 \mathrm{~min}$ and destained with a solution of $30 \%$ methanol, $7 \%$ acetic acid, and $63 \%$ water. The gel was scanned for image acquisition and the band of interest was sent to Genomine, Inc. (Pohang, South Korea) for mass spectrometry. In-gel proteolytic digestion and protein fragment extraction were conducted and results were compared with the NCBI protein database using Mascot software to identify the protein.

\section{Protein extraction}

GST and GST-hnRNP K were expressed in Escherichia coli BL21 (DE3) pLysS cells (Novagen) grown to an absorbance at $600 \mathrm{~nm}$ (A600) of 0.6. Induction of GST, GST-hnRNP K was performed by $0.5 \mathrm{mM}$ isopropyl $\beta$-D-1-thiogalactopyranoside (IPTG) overnight at $18^{\circ} \mathrm{C}$. Next, cells were resuspended in $20 \mathrm{mM}$ Tris- $\mathrm{HCl}(\mathrm{pH} 7.5), 150 \mathrm{mM} \mathrm{NaCl}, 1 \%$ Triton-X, $1 \mathrm{mM}$ dithiothreitol, and protease inhibitor cocktail (Roche) and were lysed by sonication. And, GST and GST-hnRNP K were purified using glutathione-sepharose 4B agarose beads (GE Healthcare Bio-Sciences) and were eluted in $50 \mathrm{mM}$ Tris- $\mathrm{HCl}(\mathrm{pH} 8.0)$ and $20 \mathrm{mM}$ L-Glutathione reduced (GE Healthcare).

\section{Immunoblot assay}

Immunoblotting was conducted by using polyclonal anti-hnRNP K (Abcam, ab70492), monoclonal anti-GST (Santa Cruz, sc-138), Polyclonal anti-FLAG (Cell signaling, \#2368), polyclonal anti-14-3-3 (Santa Cruz, sc-1019) and, monoclonal anti-GAPDH (Santa Cruz, sc-47724) as primary antibodies. A SUPEX ECL solution kit (Neuronex) and LAS-4000 chemiluminescence detection system (FUJIFILM) were used after horseradish peroxidase-conjugated species-specific secondary antibodies (goat, Santa Cruz Biotechnology; guinea pig, Santa Cruz Biotechnology; mouse, Thermo Scientific; rabbit, Jackson ImmunoResearch Laboratories) reactions. And, Image Gauge (Fuji Film) was used for analysis of the acquired images according to the manufacturer's instructions.

\section{MTT assay}

NIH3T3 and U2OS cells at a density of $2 \times 10^{4}$ cells per well in 96-well plates were transfected with siCon and sihnRNP K. After $48 \mathrm{hr}$, MTT $(5 \mathrm{mg} / \mathrm{ml})$ was added to each well followed by a 2-hr incubation at room temperature. Next, the media was removed and $100 \mu \mathrm{I}$ DSO was added to each well. Color development was allowed to proceed for $3 \mathrm{hr}$ at room temperature in the dark. Finally, we measured the absorbance of each well at $570 \mathrm{~nm}$ using a NanoQuant spectrophotometer (Tecan).

\section{Drosophila stocks}

Flies were raised on standard cornmeal-yeast-agar food at $25^{\circ} \mathrm{C} . w^{1118}$ (control), R18H11-Gal4, and UAS-hnRNPK $^{\text {RNAi }}$ (T42540) lines were obtained from the Bloomington Drosophila Stock Center 
(BDSC). Two UAS-hnRNPK ${ }^{\mathrm{RNAi}}$ lines (v2912, v106271) were obtained from the Vienna Drosophila RNAi Center. Pdf-Gal4 and tim-Gal4 lines were described previously. $(61,62)$

\section{Behavioral analysis}

Individual male flies were loaded into glass tubes containing 5\% sucrose and $2 \%$ agar, entrained by five 12-hour light: 12-hour dark (LD) cycles, and then transferred to constant dark (DD) for 7 days at $25^{\circ} \mathrm{C}$. Locomotor activities were continuously recorded in 1-min bins using the infrared beam-based Drosophila Activity Monitor (DAM) system (Trikinetics). Behavioral data were analyzed using the ClockLab software in MATLAB (Actimetrics). Circadian periods and the power of rhythmicity in DD locomotor rhythms were calculated in individual flies using the chi-square periodogram (the significance level was set to an alpha value of 0.05 ) and averaged per each genotype. Flies with the power of rhythmicity value less than 10 were defined as arrhythmic. Locomotor activity profiles were generated using a Microsoft Excel. Morning and evening indices anticipatory to lights-on and lights-off, respectively, were calculated as described previously (69). Dead flies were manually scored at the end of behavioral tests and their locomotor data were excluded from further analyses.

\section{Statistical analysis.}

Graphs are plotted as a mean \pm S.E.M which was illustrated by bars. Two-way ANOVA was used to conduct grouped analyses with multiple groups by Sidak's multiple comparisons test. One-way ANOVA was used to conduct column analyses with more than two groups with Tukey's multiple comparisons test analyzed with PRISM software.

\section{DATA AVAILABILITY}

hnRNP K bound genes were available in Huarte et al. 2010 (Cell, 142, 409-419). MEME (Multiple Em for Motif Elicitation), MEME-ChIP, GOMo (Gene Ontology for Motifs) algorithms were available at http://meme-suite.org/

\section{ACKNOWLEDGEMENT}

The authors thank Ze'ev Ronai (Sanford Burnham Prebys Medical Discovery Institute) for providing HA-hnRNP K WT, Jürgen A. Ripperger (University of Fribourg) for providing DBP-luciferase plasmid, and Sung-Hoon Kim (McGill University) for providing the GST-hnRNP K plasmid. This work was supported by the Bio \& Medical Technology Development Program of the National Research Foundation (NRF) funded by the Korean government (MSIT) (No. 2018011982); "Cooperative Research Program for Agriculture Science \& Technology Development (Project No. PJ01324801)" by Rural Development Administration; and BK21 Plus funded by the Ministry of Education, Republic of Korea (10Z20130012243). 


\section{Author contributions}

P. K. Kwon, K-H. Lee, J. Kim, S. Tae, H-M Kim, S. Ham, J-H Choi, Y-H Jeong, and S-W Kim conducted and analyzed the experiments; $\mathrm{H}$. Yi and $\mathrm{H}-\mathrm{O}$ Ku analyzed the lumicycle data; T-Y Roh, $\mathrm{C}$. Lim, and K-T Kim designed the experiments; P. K. Kwon and K-T Kim wrote the paper.

Conflict of interest statement. None declared

\section{Uncategorized References}

1. Yu W \& Hardin PE (2006) Circadian oscillators of Drosophila and mammals. J Cell Sci 119(Pt 23):4793-4795.

2. Lowrey PL \& Takahashi JS (2004) Mammalian circadian biology: elucidating genome-wide levels of temporal organization. Annu Rev Genomics Hum Genet 5:407-441.

3. Koike $\mathrm{N}$, et al. (2012) Transcriptional architecture and chromatin landscape of the core circadian clock in mammals. Science 338(6105):349-354.

4. Stratmann M, Suter DM, Molina N, Naef F, \& Schibler U (2012) Circadian Dbp transcription relies on highly dynamic BMAL1-CLOCK interaction with $\mathrm{E}$ boxes and requires the proteasome. Mol Cell 48(2):277-287.

5. King DP, et al. (1997) Positional cloning of the mouse circadian clock gene. Cell 89(4):641653.

6. Gekakis N, et al. (1998) Role of the CLOCK protein in the mammalian circadian mechanism. Science 280(5369):1564-1569.

7. Griffin EA, Jr., Staknis D, \& Weitz CJ (1999) Light-independent role of CRY1 and CRY2 in the mammalian circadian clock. Science 286(5440):768-771.

8. Kume K, et al. (1999) mCRY1 and mCRY2 are essential components of the negative limb of the circadian clock feedback loop. Cel/98(2):193-205.

9. Tei H, et al. (1997) Circadian oscillation of a mammalian homologue of the Drosophila period gene. Nature 389(6650):512-516.

10. Albrecht U, Sun ZS, Eichele G, \& Lee CC (1997) A differential response of two putative mammalian circadian regulators, mper1 and mper2, to light. Cel/91(7):1055-1064.

11. Sun ZS, et al. (1997) RIGUI, a putative mammalian ortholog of the Drosophila period gene. Cel/90(6):1003-1011.

12. Zylka MJ, Shearman LP, Weaver DR, \& Reppert SM (1998) Three period homologs in mammals: differential light responses in the suprachiasmatic circadian clock and oscillating transcripts outside of brain. Neuron 20(6):1103-1110.

13. Kim TD, et al. (2007) Rhythmic control of AANAT translation by hnRNP Q in circadian melatonin production. Genes Dev21(7):797-810.

14. Lim I, Jung Y, Kim DY, \& Kim KT (2016) HnRNP Q Has a Suppressive Role in the Translation 
of Mouse Cryptochrome1. PLoS One 11(7):e0159018.

15. Lee $\mathrm{KH}$, et al. (2012) Rhythmic interaction between Period1 mRNA and hnRNP Q leads to circadian time-dependent translation. Mol Cell Bio/32(3):717-728.

16. Kim DY, Woo KC, Lee KH, Kim TD, \& Kim KT (2010) hnRNP Q and PTB modulate the circadian oscillation of mouse Rev-erb alpha via IRES-mediated translation. Nucleic Acids Res 38(20):7068-7078.

17. Woo KC, et al. (2010) Circadian amplitude of cryptochrome 1 is modulated by mRNA stability regulation via cytoplasmic hnRNP D oscillation. Mol Cell Bio/30(1):197-205.

18. Kim HJ, et al. (2017) Heterogeneous nuclear ribonucleoprotein A1 regulates rhythmic synthesis of mouse Nfil3 protein via IRES-mediated translation. Sci Rep 7:42882.

19. Lee HR, et al. (2015) Heterogeneous ribonucleoprotein $\mathrm{R}$ regulates arylalkylamine $\mathrm{N}$ acetyltransferase synthesis via internal ribosomal entry site-mediated translation in a circadian manner. J Pineal Res 59(4):518-529.

20. Kim SH, et al. (2015) Rhythmic control of mRNA stability modulates circadian amplitude of mouse Period3 mRNA. J Neurochem 132(6):642-656.

21. Yang S, Van Dongen HP, Wang K, Berrettini W, \& Bucan M (2009) Assessment of circadian function in fibroblasts of patients with bipolar disorder. Mol Psychiatry 14(2):143-155.

22. Luo WY, et al. (2012) Old flies have a robust central oscillator but weaker behavioral rhythms that can be improved by genetic and environmental manipulations. Aging Cell11(3):428-438.

23. Gloston GF, Yoo SH, \& Chen ZJ (2017) Clock-Enhancing Small Molecules and Potential Applications in Chronic Diseases and Aging. Front Neuro/8:100.

24. Chen $\mathrm{CY}$, et al. (2016) Effects of aging on circadian patterns of gene expression in the human prefrontal cortex. Proc Natl Acad Sci U S A 113(1):206-211.

25. Wuarin J \& Schibler U (1990) Expression of the liver-enriched transcriptional activator protein DBP follows a stringent circadian rhythm. Cel/63(6):1257-1266.

26. Mueller CR, Maire P, \& Schibler U (1990) DBP, a liver-enriched transcriptional activator, is expressed late in ontogeny and its tissue specificity is determined posttranscriptionally. Cell 61(2):279-291.

27. Wuarin J, et al. (1992) The role of the transcriptional activator protein DBP in circadian liver gene expression. J Cell Sci Supp/16:123-127.

28. Ueda HR, et al. (2005) System-level identification of transcriptional circuits underlying mammalian circadian clocks. Nat Genet37(2):187-192.

29. Mitsui S, Yamaguchi S, Matsuo T, Ishida Y, \& Okamura H (2001) Antagonistic role of E4BP4 and PAR proteins in the circadian oscillatory mechanism. Genes Dev 15(8):995-1006.

30. Falvey E, Marcacci L, \& Schibler U (1996) DNA-binding specificity of PAR and C/EBP leucine zipper proteins: a single amino acid substitution in the C/EBP DNA-binding domain confers PAR-like specificity to C/EBP. Biol Chem 377(12):797-809.

31. Kumaki $\mathrm{Y}$, et al. (2008) Analysis and synthesis of high-amplitude Cis-elements in the mammalian circadian clock. Proc Natl Acad Sci U S A 105(39):14946-14951.

32. Bozek K, et al. (2009) Regulation of Clock-Controlled Genes in Mammals. Plos One 4(3). 
33. Ripperger JA, Shearman LP, Reppert SM, \& Schibler U (2000) CLOCK, an essential pacemaker component, controls expression of the circadian transcription factor DBP. Genes Dev 14(6):679-689.

34. Ripperger JA \& Schibler U (2006) Rhythmic CLOCK-BMAL1 binding to multiple E-box motifs drives circadian Dbp transcription and chromatin transitions. Nat Genet 38(3):369-374.

35. Stratmann M, Stadler F, Tamanini F, van der Horst GT, \& Ripperger JA (2010) Flexible phase adjustment of circadian albumin D site-binding protein (DBP) gene expression by CRYPTOCHROME1. Genes Dev24(12):1317-1328.

36. Matunis MJ, Michael WM, \& Dreyfuss G (1992) Characterization and primary structure of the poly $(\mathrm{C})$-binding heterogeneous nuclear ribonucleoprotein complex $\mathrm{K}$ protein. Mol Cell Biol 12(1):164-171.

37. Bomsztyk K, Denisenko O, \& Ostrowski J (2004) hnRNP K: one protein multiple processes. Bioessays 26(6):629-638.

38. Michelotti EF, Michelotti GA, Aronsohn Al, \& Levens D (1996) Heterogeneous nuclear ribonucleoprotein K is a transcription factor. Mol Cell Biol 16(5):2350-2360.

39. Shnyreva M, Schullery DS, Suzuki H, Higaki Y, \& Bomsztyk K (2000) Interaction of two multifunctional proteins. Heterogeneous nuclear ribonucleoprotein $\mathrm{K}$ and $\mathrm{Y}$-box-binding protein. J Bio/ Chem 275(20):15498-15503.

40. Tomonaga T \& Levens D (1996) Activating transcription from single stranded DNA. Proc Natl Acad Sci U S A 93(12):5830-5835.

41. Michelotti GA, et al. (1996) Multiple single-stranded cis elements are associated with activated chromatin of the human c-myc gene in vivo. Mol Cell Biol 16(6):2656-2669.

42. Banerjee $\mathrm{K}$, et al. (2014) Regulation of tyrosine hydroxylase transcription by hnRNP K and DNA secondary structure. Nat Commun 5:5769.

43. Rey G, et al. (2011) Genome-wide and phase-specific DNA-binding rhythms of BMAL1 control circadian output functions in mouse liver. PLOS Bio/9(2):e1000595.

44. Kiyohara YB, et al. (2008) Detection of a circadian enhancer in the mDbp promoter using prokaryotic transposon vector-based strategy. Nucleic Acids Res 36(4):e23.

45. Yamajuku D, et al. (2010) Identification of functional clock-controlled elements involved in differential timing of Per1 and Per2 transcription. Nucleic Acids Res 38(22):7964-7973.

46. Zhang L, Ding Q, \& Wang Z (2012) Nuclear respiratory factor 1 mediates the transcription initiation of insulin-degrading enzyme in a TATA box-binding protein-independent manner. PLoS One 7(8):e42035.

47. Huarte $\mathrm{M}$, et al. (2010) A large intergenic noncoding RNA induced by $\mathrm{p} 53$ mediates global gene repression in the p53 response. Cell 142(3):409-419.

48. Braddock DT, Baber JL, Levens D, \& Clore GM (2002) Molecular basis of sequence-specific single-stranded DNA recognition by $\mathrm{KH}$ domains: solution structure of a complex between hnRNP K KH3 and single-stranded DNA. EMBO J21(13):3476-3485.

49. Choi HS, et al. (2009) Poly $(\mathrm{C})$-binding proteins as transcriptional regulators of gene expression. Biochem Biophys Res Commun 380(3):431-436. 
50. Dickey TH, Altschuler SE, \& Wuttke DS (2013) Single-stranded DNA-binding proteins: multiple domains for multiple functions. Structure 21(7):1074-1084.

51. Habelhah H, et al. (2001) ERK phosphorylation drives cytoplasmic accumulation of hnRNP-K and inhibition of mRNA translation. Nat Cell Bio/3(3):325-330.

52. Ko GY, Ko ML, \& Dryer SE (2001) Circadian regulation of cGMP-gated cationic channels of chick retinal cones. Erk MAP Kinase and Ca2+/calmodulin-dependent protein kinase II. Neuron 29(1):255-266.

53. Goldsmith CS \& Bell-Pedersen D (2013) Diverse roles for MAPK signaling in circadian clocks. Adv Genet 84:1-39.

54. Hovemann BT, Reim I, Werner S, Katz S, \& Saumweber H (2000) The protein Hrb57A of Drosophila melanogaster closely related to hnRNP $\mathrm{K}$ from vertebrates is present at sites active in transcription and coprecipitates with four RNA-binding proteins. Gene 245(1):127137.

55. Charroux B, Angelats C, Fasano L, Kerridge S, \& Vola C (1999) The levels of the bancal product, a Drosophila homologue of vertebrate hnRNP K protein, affect cell proliferation and apoptosis in imaginal disc cells. Mol Cell Biol 19(11):7846-7856.

56. Gates J \& Thummel CS (2000) An enhancer trap screen for ecdysone-inducible genes required for Drosophila adult leg morphogenesis. Genetics 156(4):1765-1776.

57. Zhang L, et al. (2010) DN1(p) circadian neurons coordinate acute light and PDF inputs to produce robust daily behavior in Drosophila. Curr Biol20(7):591-599.

58. Zhang Y, Liu Y, Bilodeau-Wentworth D, Hardin PE, \& Emery P (2010) Light and temperature control the contribution of specific DN1 neurons to Drosophila circadian behavior. Curr Biol 20(7):600-605.

59. Kunst $M$, et al. (2014) Calcitonin gene-related peptide neurons mediate sleep-specific circadian output in Drosophila. Curr Bio/24(22):2652-2664.

60. Flourakis $M$, et al. (2015) A Conserved Bicycle Model for Circadian Clock Control of Membrane Excitability. Cel/162(4):836-848.

61. Kaneko M \& Hall JC (2000) Neuroanatomy of cells expressing clock genes in Drosophila: transgenic manipulation of the period and timeless genes to mark the perikarya of circadian pacemaker neurons and their projections. J Comp Neuro/ 422(1):66-94.

62. Renn SC, Park JH, Rosbash M, Hall JC, \& Taghert PH (1999) A pdf neuropeptide gene mutation and ablation of PDF neurons each cause severe abnormalities of behavioral circadian rhythms in Drosophila. Cel/99(7):791-802.

63. Sobel JA, et al. (2017) Transcriptional regulatory logic of the diurnal cycle in the mouse liver. PLOS Biol 15(4):e2001069.

64. Machanick P \& Bailey TL (2011) MEME-ChIP: motif analysis of large DNA datasets. Bioinformatics 27(12):1696-1697.

65. Lee $\mathrm{KH}$, et al. (2014) AUF1 contributes to Cryptochrome1 mRNA degradation and rhythmic translation. Nucleic Acids Res 42(6):3590-3606.

66. Ran FA, et al. (2013) Genome engineering using the CRISPR-Cas9 system. Nat Protoc 
8(11):2281-2308.

67. Corces MR, et al. (2017) An improved ATAC-seq protocol reduces background and enables interrogation of frozen tissues. Nat Methods 14(10):959-+.

68. Buenrostro JD, Giresi PG, Zaba LC, Chang HY, \& Greenleaf WJ (2013) Transposition of native chromatin for fast and sensitive epigenomic profiling of open chromatin, DNA-binding proteins and nucleosome position. Nat Methods 10(12):1213-+.

69. Seluzicki A, et al. (2014) Dual PDF signaling pathways reset clocks via TIMELESS and acutely excite target neurons to control circadian behavior. PLOS Bio/12(3):e1001810. 


\section{Figure Legends}

Figure 1. Oscillation of DBP mRNA expression is supported by its transcription at specific promoter regions. (A) Promoter assay was conducted at $24 \mathrm{hrs}$ after Dexamethasone synchronization. P3, P4, P6, WT, and WT(Long) promoter regions showed significant promoter activities, which suggested that the region between -300 and -400 from the TSS was a critical promoter region. In contrast, other promoter regions had relatively little impact on promoter activity. $(n=5$, indicated by asterisks; * $p<0.05$ by Tukey's multiple comparisons test). (B) Representative baseline detrended bioluminescence recordings from WT:LUC, P2:LUC, and P3:LUC, which was transiently transfected into an NIH3T3 cell line that is synchronized by dexamethasone, suggested that the regions between -300 and -400 from the TSS could be crucial in high-amplitude of DBP oscillation. (C) Poly $(C)$ motif (or CCT motif) was scanned in the region between -300 and -400 from TSS using the MEME suite. * indicates the region where poly $(C)$ motif is. (D) One of the predicted functions of poly $(C)$ motif by GOMo was the transcription. Poly $(C)$ motif was the only motif that was predicted to function in transcription among motifs. MF; Molecular function, BP; Biological process. (E) Alignment of genomic regions containing a pair of poly $(C)$ motif showed high conservation of the motif among eutherian mammals, which suggests that a common transcriptional regulatory mechanism is involved in the DBP promoter activity. Underline and bold font represent poly(C) motifs. (F) Illustration of the luciferase reporter system containing WT promoter and poly $(\mathrm{C})$ mutant is described. Bold font indicates poly $(\mathrm{C})$ motif. Underline and italic font represent mutated bases. (G) Promoter activity at $24 \mathrm{hrs}$ after Dexamethasone synchronization of the poly $(\mathrm{C})$ mutant showed a significant decrease compared to WT, which suggests that poly $(\mathrm{C})$ motif is crucial for promoter activity ( $n=3$, Indicated by asterisks; ${ }^{*} p<0.05$ by Unpaired t-test). $(H)$ Representative baseline detrended bioluminescence recordings of WT:LUC and poly $(\mathrm{C})$ mutant:LUC were measured with luciferase reporter transfected NIH3T3 cell line after dexamethasone synchronization, which showed a decrease in the amplitude level of the poly $(C)$ mutant.

Figure 2. The poly $(C)$ motif is critical for high-amplitude DBP mRNA oscillation. (A) Schematic description of a pair of sgRNAs designed to delete endogenous poly $(C)$ motif regions within the DBP proximal promoter region is shown. $(B) \operatorname{Poly}(\mathrm{C})$ motif region deleted cell line made through CRISPRCas9 system was confirmed by sequencing. (C) The oscillation of endogenous DBP mRNA was quantified and the poly $(C)$ motif region deleted cell line showed a decrease in amplitude of DBP mRNA oscillation ( $n=4$, Error bar: S.E.M.). (D) Per2 mRNA oscillation level was quantified, which oscillation was affected by the poly $(C)$ motif within the DBP promoter ( $n=5$, Error bar: S.E.M.). (E) Cry1 mRNA oscillation was measured. The phase of Cry1 mRNA oscillation was slightly changed ( $n=4$, Error bar: S.E.M.). (F) Clock mRNA oscillation was used as a control ( $n=5$, Error bar: S.E.M.). (G) Pol2 ChIP was conducted to measure the recruitment level of Pol2 to DBP promoter regions at the peak time of DBP mRNA level ( $n=3$, indicated by asterisks; * $p<0.05$ by Tukey's multiple comparisons test). (H) ATACqPCR data indicated that chromatin accessibility of WT cells increased at $24 \mathrm{hrs}$ after Dexamethasone synchronization, however, poly $(C)$ motif region deleted cells did not show an increase in the chromatin 
accessibility ( $n=4$, n.s. : not significant, indicated by asterisks; * $p<0.05$ by Tukey's multiple comparisons test).

Figure 3. hnRNP $K$ binds to single-stranded poly(C) motif directly and rhythmically. (A) Coomassie staining showed that one thick band was bound onto the forward poly $(C)$ motif oligonucleotide, which was identified as hnRNP $\mathrm{K}$ by Mass spectrometry. In contrast, there were no prominent proteins detected at complementary poly(C) motif oligonucleotide. (B) Purification of GST and GST-hnRNP $\mathrm{K}$ was also carried out for GST pull-down assay. GST-hnRNP K was detected at wildtype oligonucleotide, but not at the mutant oligonucleotide, which demonstrates that hnRNP $\mathrm{K}$ is directly bound to poly $(C)$ motif. $(C)$ The schematic description of the motif analysis is shown. Proximal promoter regions of 1056 genes were analyzed using the database from M. Huarte et al. 2010. Motif analysis showed their poly $(\mathrm{C})$ motif was frequently found along with a CCT motif. (D) Endogenous binding of hnRNP $\mathrm{K}$ on proximal promoter regions of DBP was confirmed by using ChIP assay. R1, R2, and R3 indicate each genomic regions. RB1 was used as a positive control, while Gapdh was used as a negative control ( $n=3$, indicated by asterisks; ${ }^{*} p<0.05$ by Tukey's multiple comparisons test). (E) Rhythmic binding of hnRNP $\mathrm{K}$ was confirmed through triggering mRNA oscillation in NIH3T3 cells. PremRNA levels of DBP showed the proportional relationship between transcription of DBP and the binding levels of hnRNP K. Relative percent input (\%) indicates the percent input (\%) level normalized by the Gapdh percent input (\%) level ( $n=3$, Error bar: S.E.M.). (F) The rhythmic expression of the nuclear hnRNP K protein could be a major contributing factor of rhythmic binding of hnRNP K. After triggering the circadian rhythm by dexamethasone synchronization, the levels of nuclear hnRNP $K$ and cytoplasmic hnRNP K were measured after the subcellular fractionation. Pol2 was used as a nuclear marker gene and GAPDH was used as a cytoplasmic marker gene. (G) The nuclear protein levels and cytoplasmic protein levels of hnRNP K were quantified ( $n=5$, Error bar: S.E.M.). (H) Wild-type and phosphomutant hnRNP K overexpression before times after Dexamethasone synchronization indicated that mutant hnRNP $\mathrm{K}$ raised DBP mRNA more than wild-type hnRNP $\mathrm{K}$ at the peak time of ERK activation level ( $n=3$, Indicated by asterisks; ${ }^{*} p<0.05$ by Unpaired t-test).

Figure 4. hnRNP K expression level controls the transcription of DBP through the poly(C) motif. (A) Knockdown of hnRNP $\mathrm{K}$ was mediated by siRNA transfection in NIH3T3 cells. The group with hnRNP K siRNA pool transfection showed a significant decrease of the hnRNP K protein level $(n=5$, indicated by asterisks; * $p<0.05$ by Unpaired t-test). (B) MTT assay showed that the knockdown of hnRNP K did not significantly affect the cell viability of NIH3T3 cell line ( $n=3$, n.s., not significant; $p<0.05$ by Unpaired t-test). (C) Along with WT and WT(Long), the level of DBP-luciferase which contained regulatory motifs within exons and introns was similarly downregulated by the lack of hnRNP $K$ ( $n=3$, indicated by asterisks; * $p<0.05$ by Sidak's multiple comparisons test). (D) Knockdown of hnRNP K decreased the level of P3, P4, P6, and WT promoter activity which contained the poly $(C)$ motif regions ( $n=3$, n.s. : not significant, indicated by asterisks; * $p<0.05$ by Tukey's multiple 
comparisons test). (E) To identify the effect of knockdown of hnRNP K on WT and poly(C) mutant, promoter assay was conducted with siRNA transfection. It showed a significant decrease only with WT promoter activity ( $n=3$, n.s. : not significant, indicated by asterisks; * $p<0.05$ by One-way ANOVA). (F) Overexpression of hnRNP K increased a WT promoter activity, whereas the promoter activity of the mutant was not altered $(n=5$, n.s. : not significant, indicated by asterisks; * $p<0.05$ by One-way ANOVA).

Figure 5. hnRNP K supports high-amplitude DBP oscillation by influencing transcription, not mRNA stability. (A) Pre-mRNA and mature mRNA levels of DBP were measured during knockdown of hnRNP K. Both mRNA levels of DBP were decreased. Per3 was used as a positive control. $(n=3$, indicated by asterisks; * $\mathrm{p}<0.05$ by Unpaired t-test). (B) During knockdown of hnRNP K, mRNA decay rates of DBP was not altered when transcription inhibitor, actinomycin D, was treated ('siCon' group : $y=-14.71 x+91.323$, 'sihnRNP K' group : $y=-14.603 x+91.192, n=3$, Error bar : S.E.M.). (C) Oscillation of DBP mRNA of mouse NIH3T3 cells was measured after dexamethasone synchronization. hnRNP K knockdown group showed a decrease in mRNA amplitude of DBP ( $n=3$, Error bar: S.E.M.). (D) Dexamethasone synchronized human U2OS cells also showed a decrease in amplitude of DBP during the knockdown of hnRNP K ( $n=3$, Error bar: S.E.M.). (E-G) Per1, Per2, and, Clock mRNA oscillation levels were quantified ( $n=3$, Error bar: S.E.M.). (H-J) Clock ('siCon' group : $y=-6.0883 x+$ 97.896, 'sinnRNP K' group : $y=-8.9137 x+98.99, n=3$, Error bar : S.E.M.), Per1 ('siCon' group : $y=-$ $0.1319 x+0.8176$, 'sihnRNP K' group : $y=-0.1291 x+0.8196, n=4$, Error bar : S.E.M.), and Per2 ('siCon' group : $y=-0.1478 x+0.8497$, 'sihnRNP K' group : $y=-0.1486 x+0.8144, n=4$, Error bar : S.E.M.) mRNA decay kinetics were measured. It indicated that Clock mRNA stability might be controlled by hnRNP K, however, Per1 and Per2 mRNA were not or marginal.

Figure 6. Drosophila hnRNP $K$ in DN1p neurons sustains circadian behaviors. (A) hnRNP K depletion in DN1p neurons affected circadian locomotor activities in both LD (12-hour light: 12-hour dark) and DD (constant dark) cycles. Normalized activity profiles in LD cycles (top) or in the first DD cycle (middle) were averaged from individual male flies $(n=26-54)$ while their averaged actograms were double-plotted (bottom). Arrows indicate gradual increases in locomotor activities anticipatory to lightson or lights-off, which were quantified by morning or evening index values, respectively (average +/SEM). ZT, zeitgeber time; CT, circadian time; white/black bars, LD cycles; gray/black bars, DD cycles. (Error bar: S.E.M.). (B) Circadian periods in DD locomotor rhythms were averaged from rhythmic flies $(P-S>10)(\mathrm{n}=24-53)$. (C) The power of rhythmicity in DD locomotor rhythms was determined by the chisquared periodograms of individual flies and were averaged per each genotype ( $n=26-54)$ (n.s., not significant; ${ }^{*} P<0.05$ to both heterozygous controls as determined by one-way ANOVA, Tukey post hoc test. Error bar: S.E.M.). 
Fig 1.

A

chr7:45703747-45705246

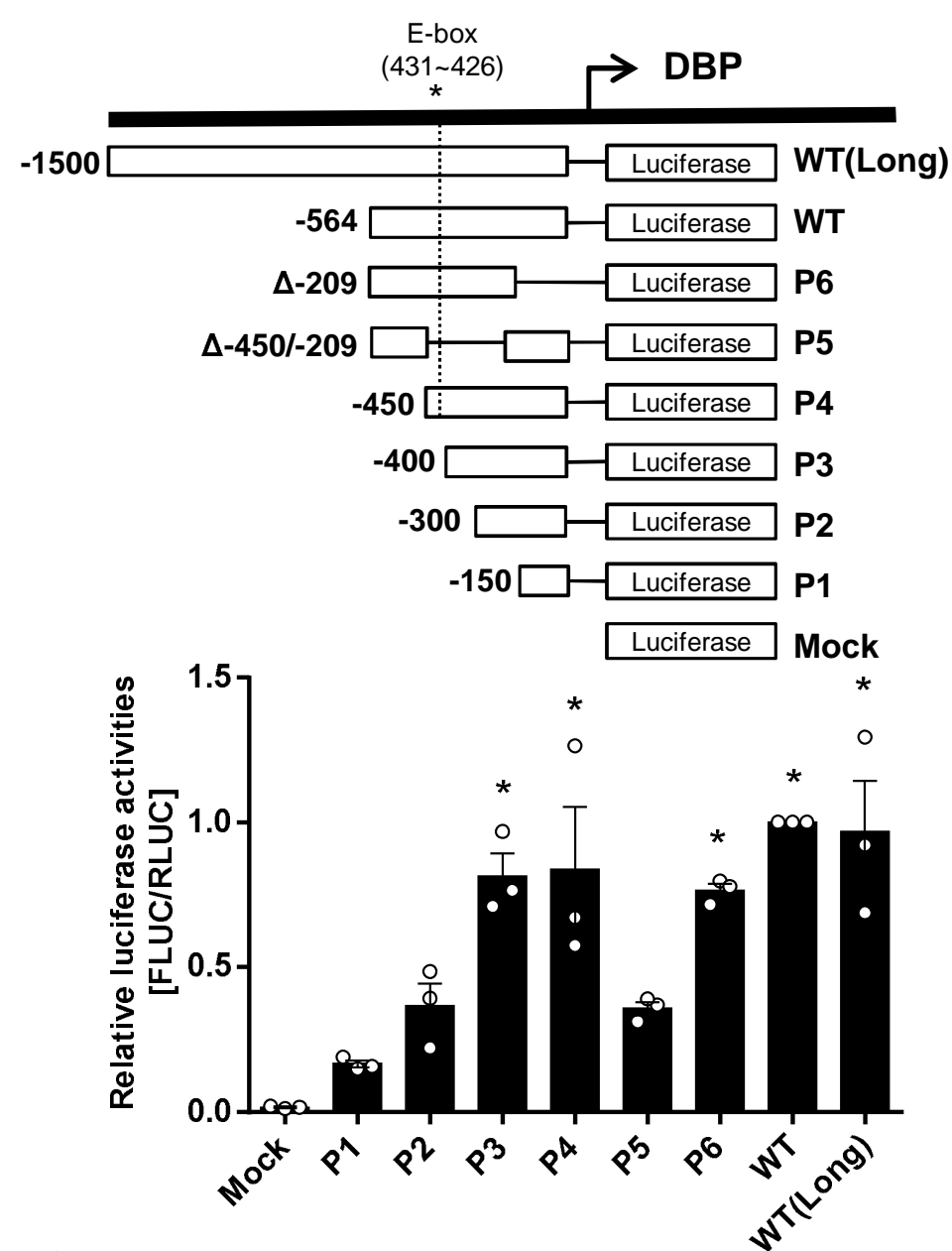

C

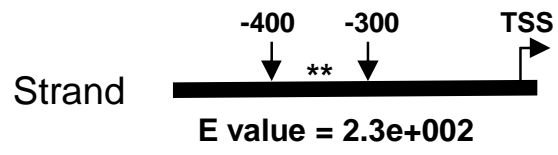

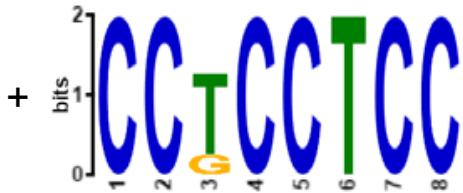

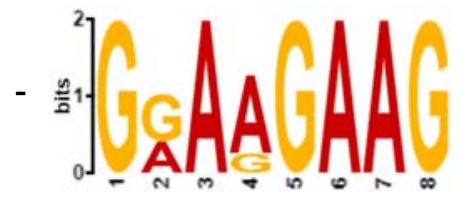

$\mathrm{F}$

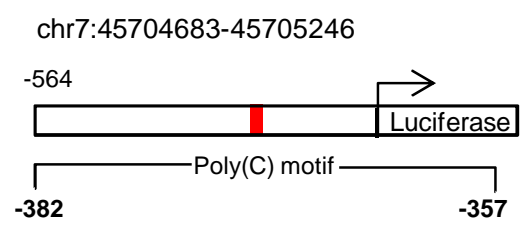

WT GTCCCGCCTCCAGCGCCTCCTCCCAT poly(C)mutant GTCAGGAATATAGCGATTCATT吕CAT

$\mathrm{G}$
B
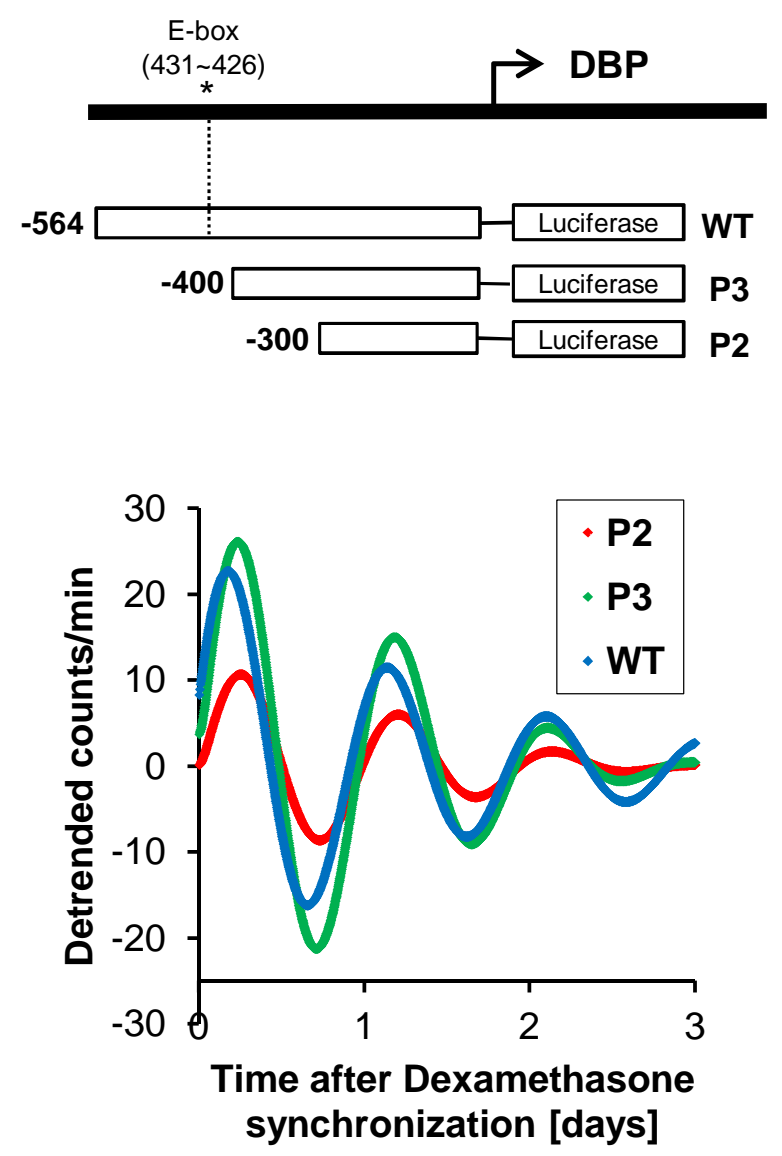

E
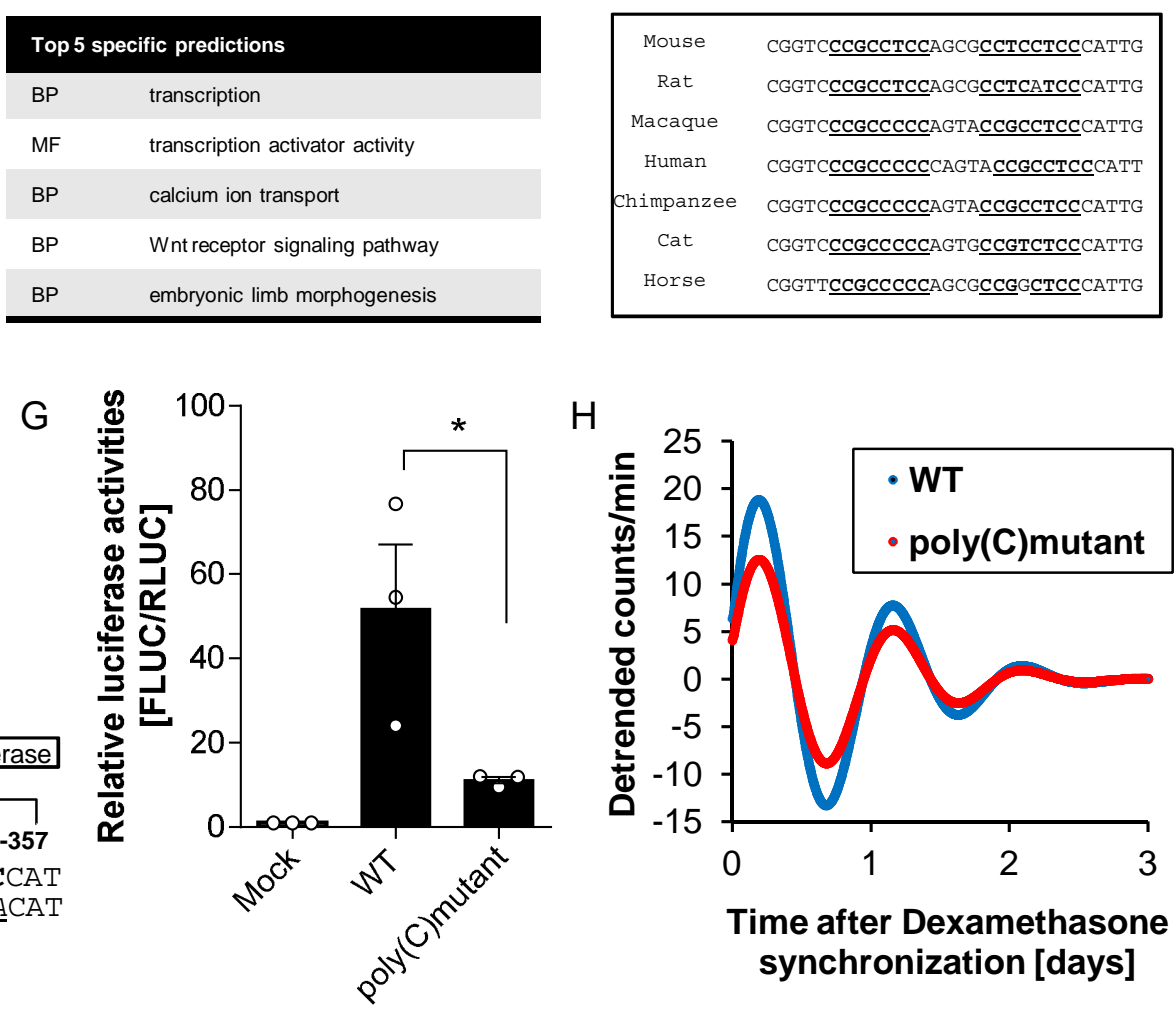

Time after Dexamethasone synchronization [days] 
Fig 2.

A

C

Target (top strand) PAM

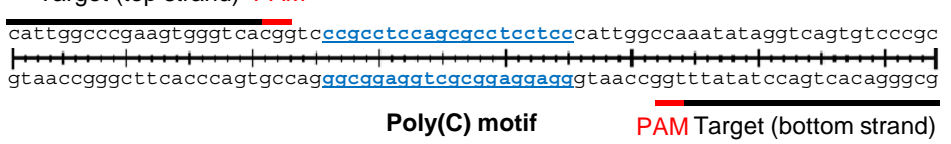

B

WT

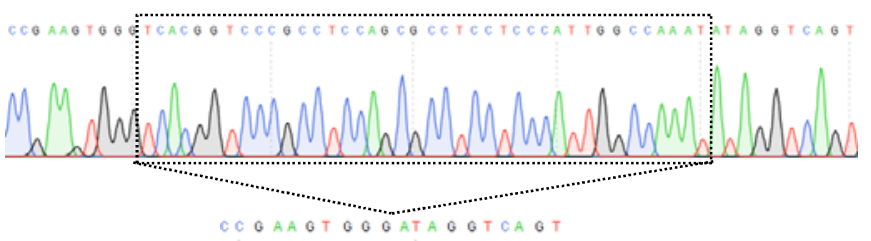

$\Delta \operatorname{poly}(\mathrm{C})$ motif

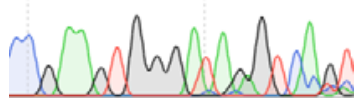

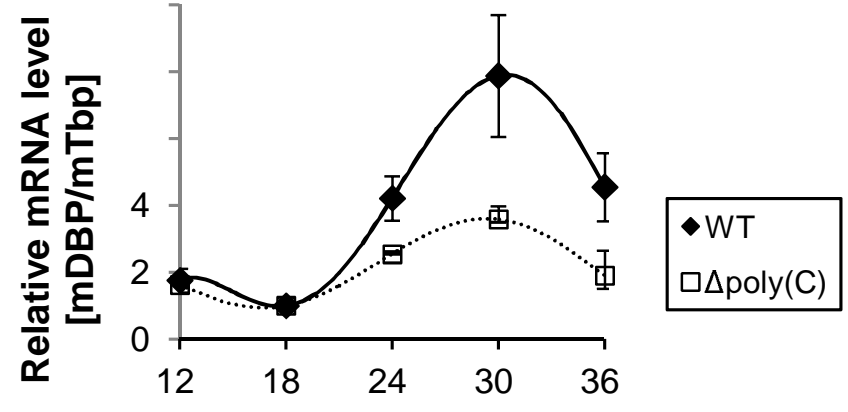

Time after Dexamethasone synchronization [hr]
D

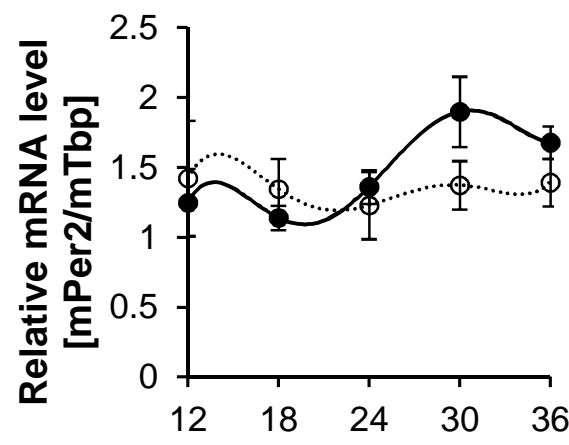

Time after Dexamethasone synchronization [hr]

G

Pol2 ChIP

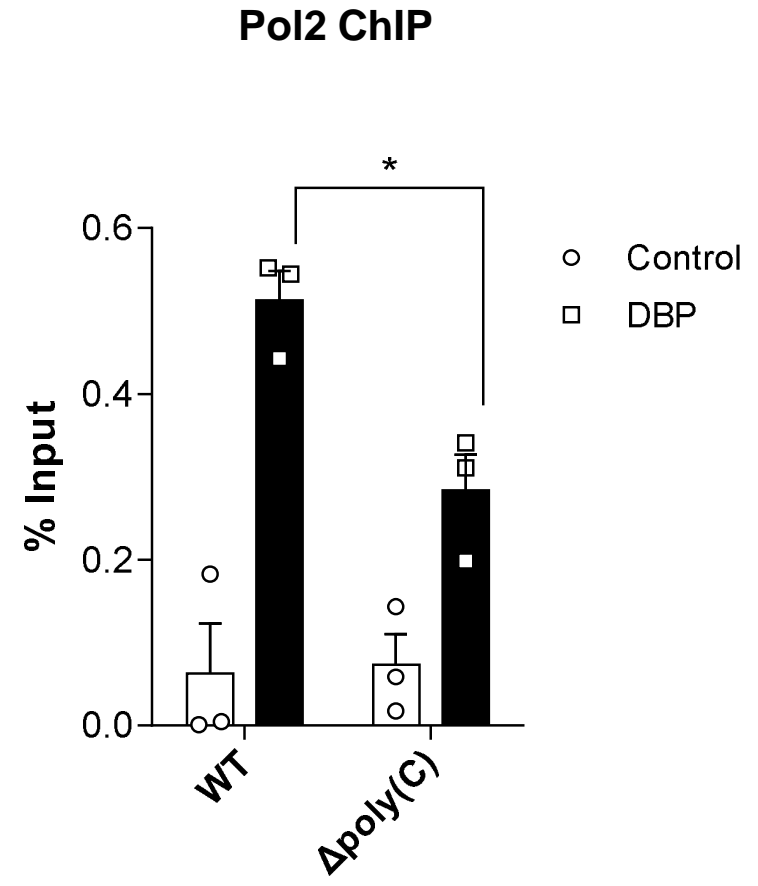

F

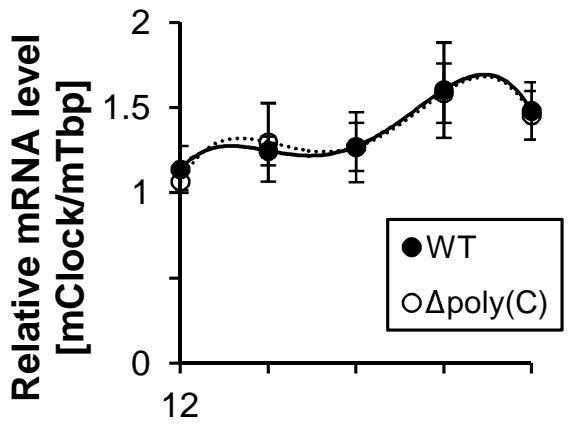

Time after Dexamethasone synchronization [hr]

$\mathrm{H}$

\section{ATAC-qPCR}
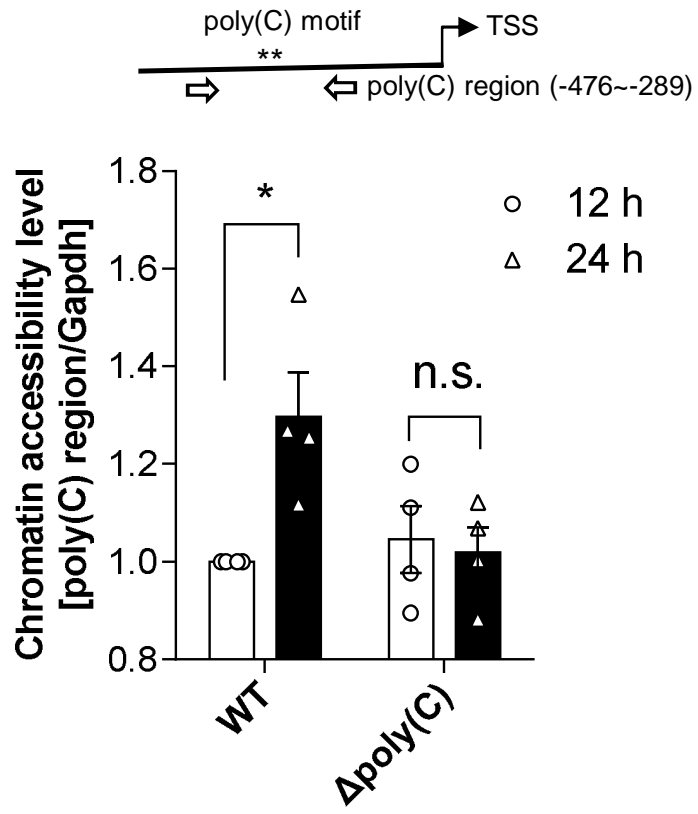
Fig 3.

A

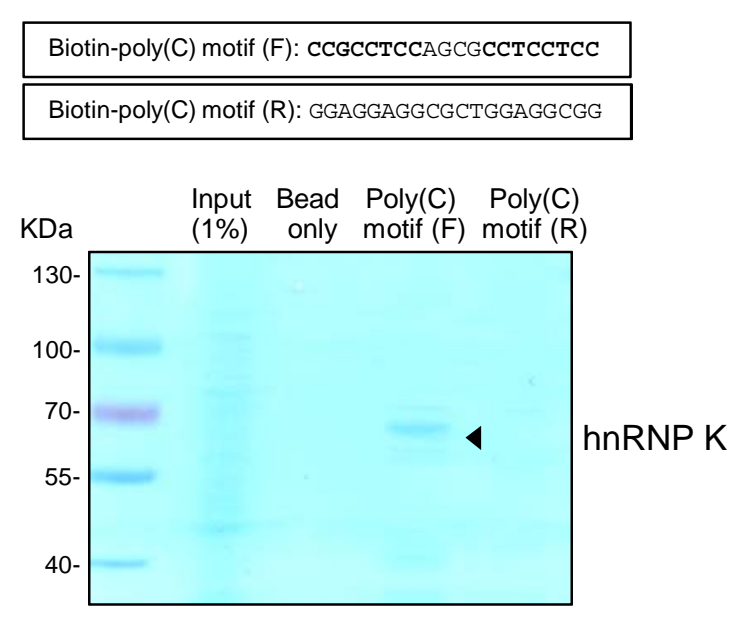

C

hnRNP K-bound promoters (1056 genes) M. Huarte et al. 2010

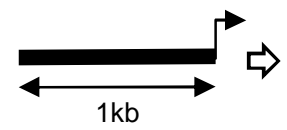

Extract sequences

of proximal promoter regions

( 1 kb from TSS)
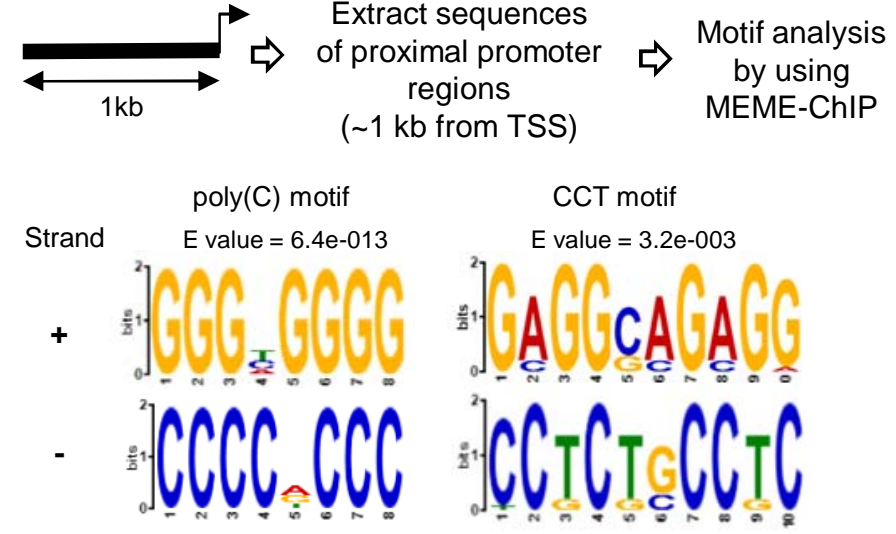

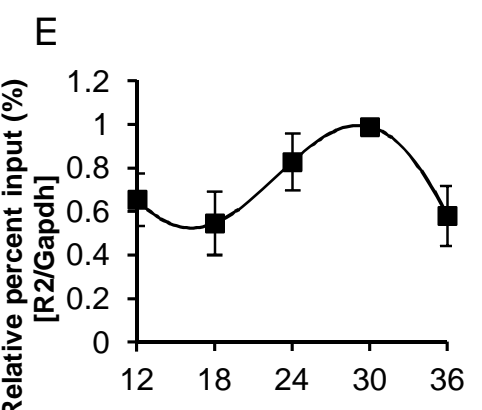

Times after Dexamethasone synchronization [hr]

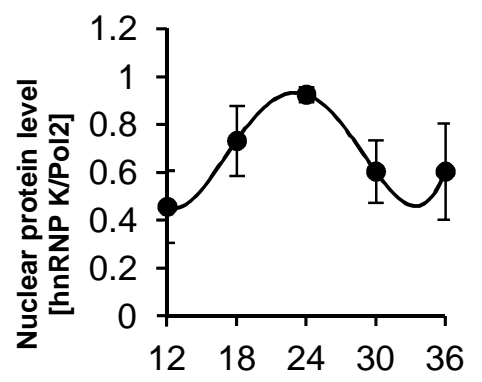

Time after Dexamethasone synchronization [hr]

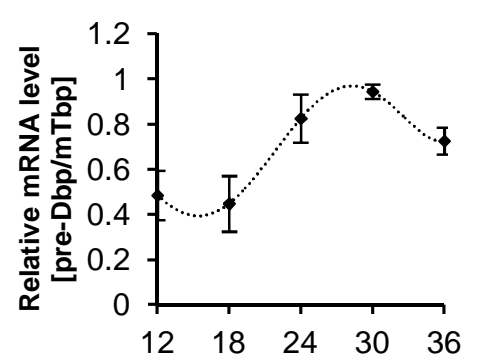

Time after Dexamethasone synchronization [hr]

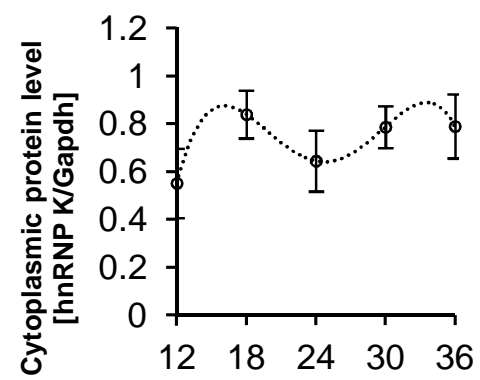

Time after Dexamethasone synchronization [hr]

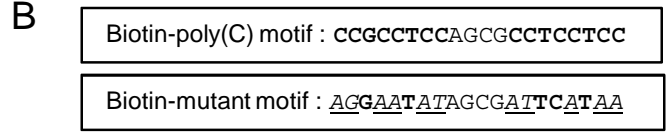

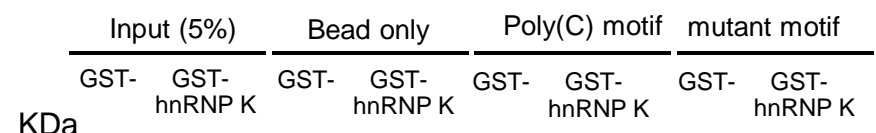

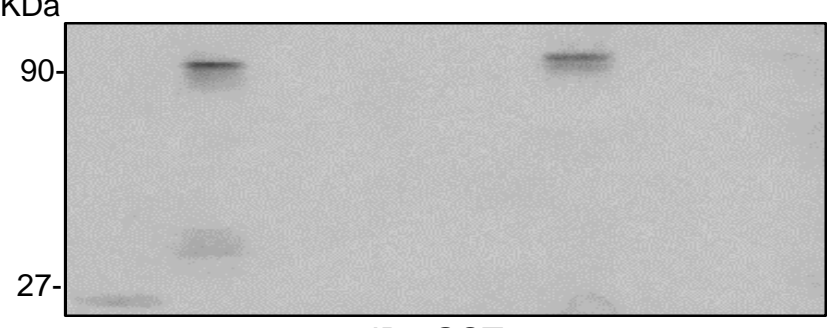

D

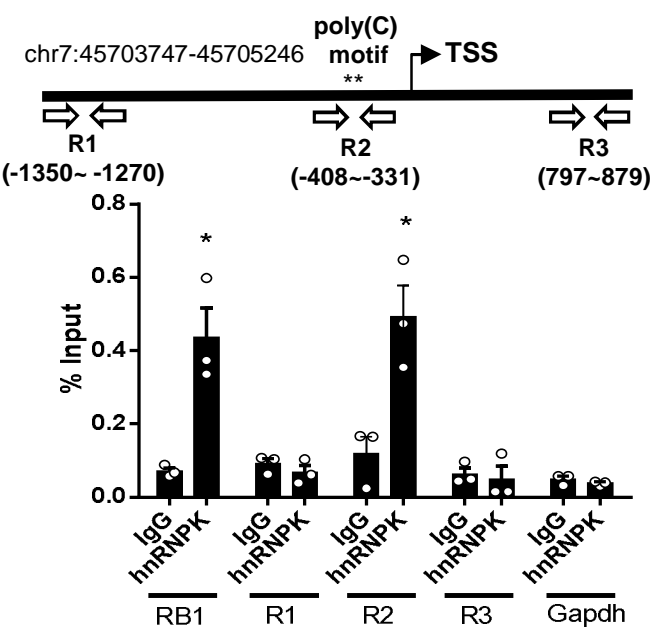

F

Dex. (hr)

IB : hnRNP K

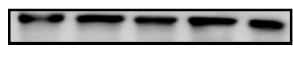

IB : GAPDH
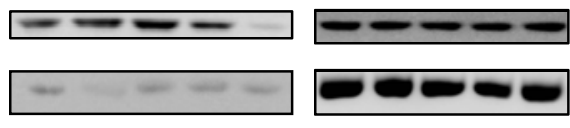

$\mathrm{H}$
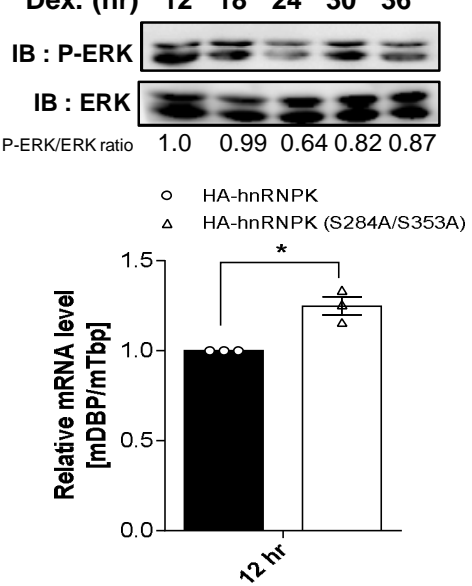
Fig 4.

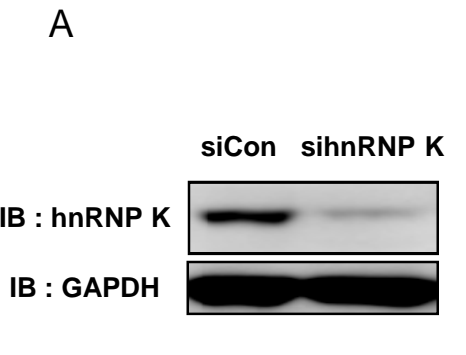

C

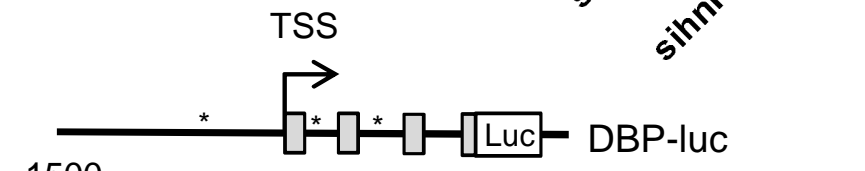

$-1500$

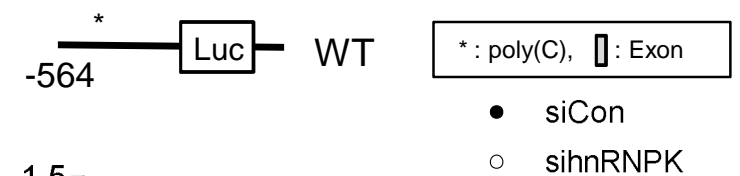

E
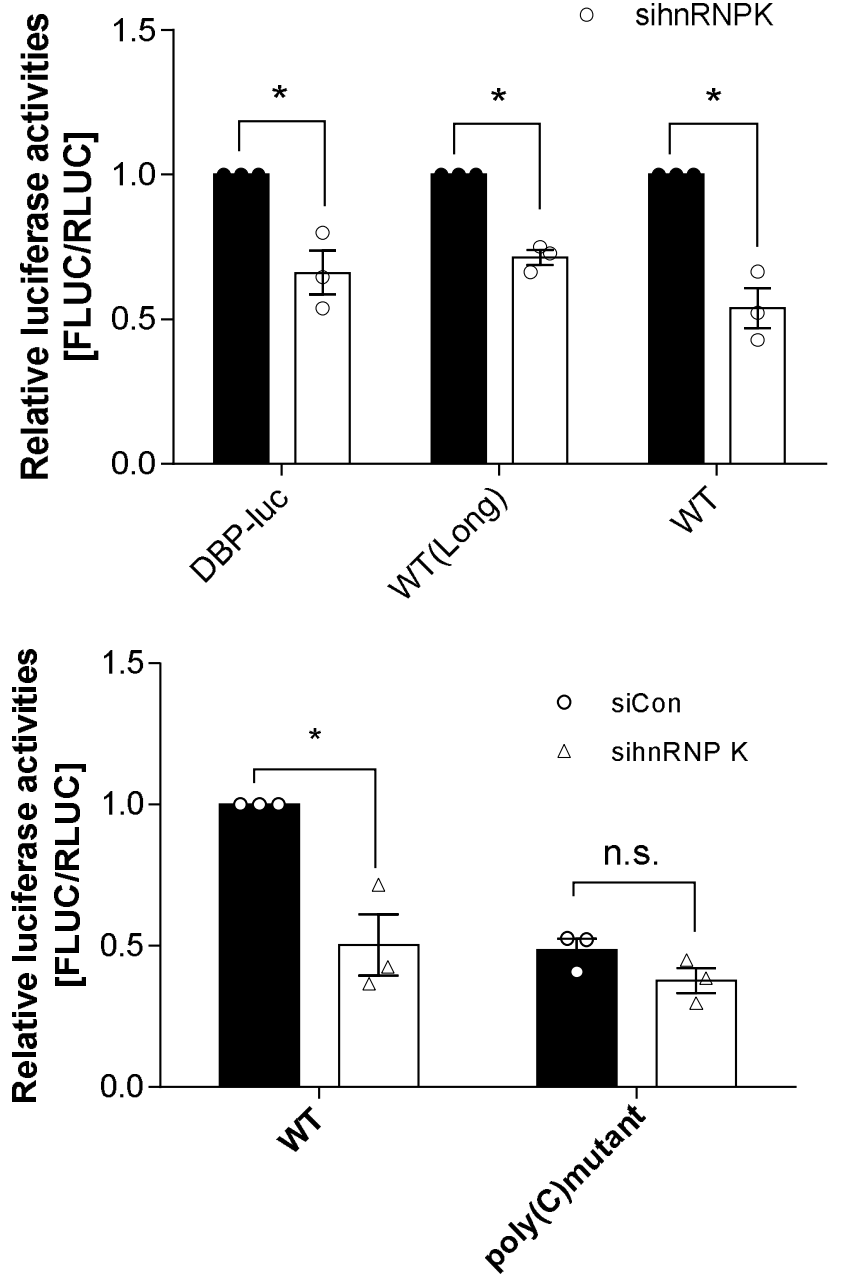

$\mathrm{B}$

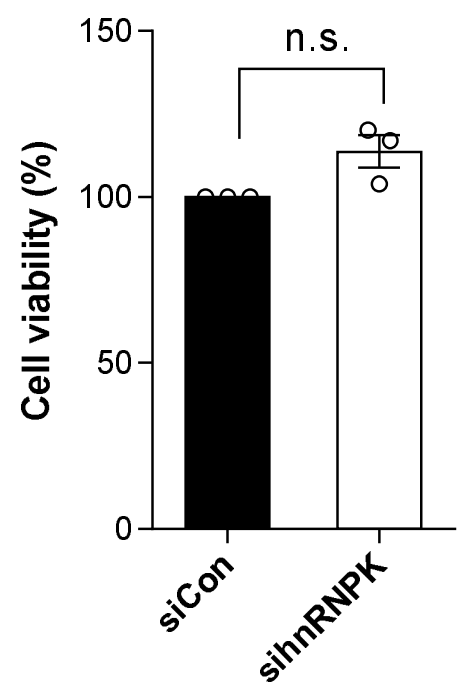

- siCon

- sihnRNPK

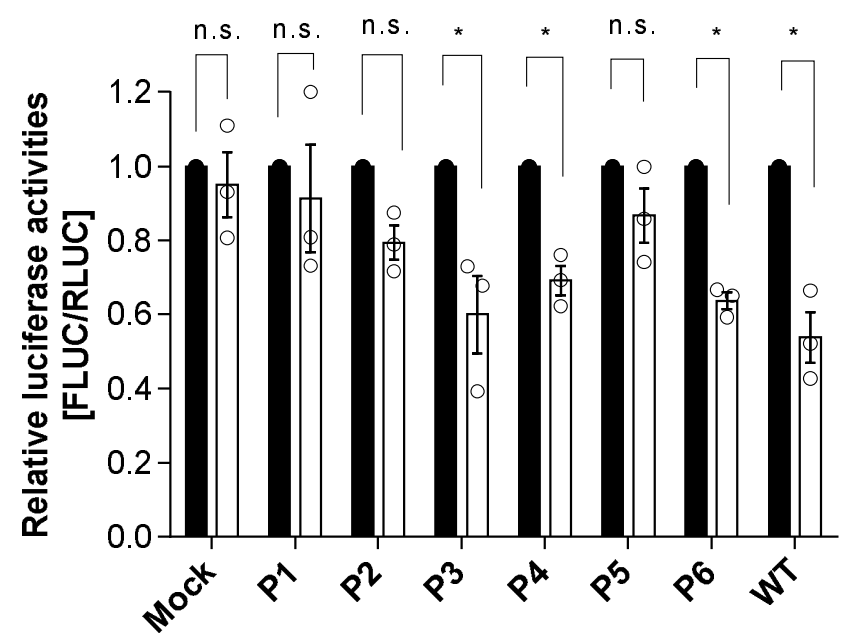

$\mathrm{F}$

HA HA-hnRNP K
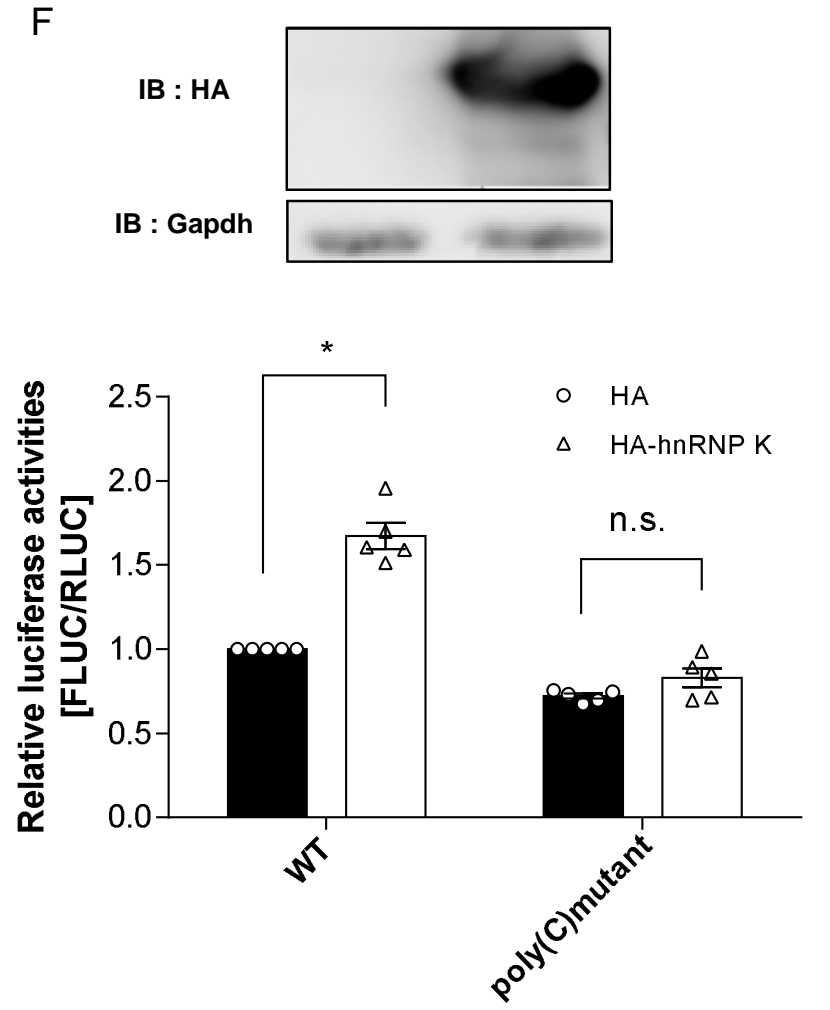
Fig 5.

- siCon

- sihnRNPK

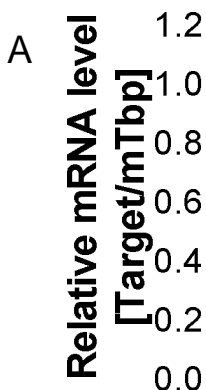

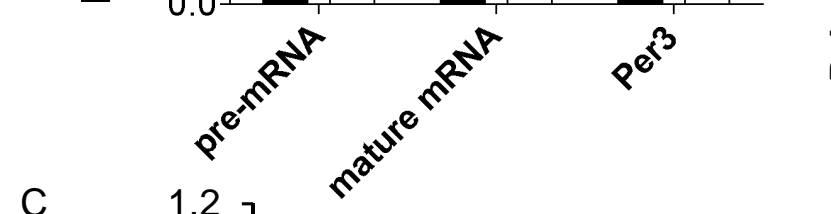

C

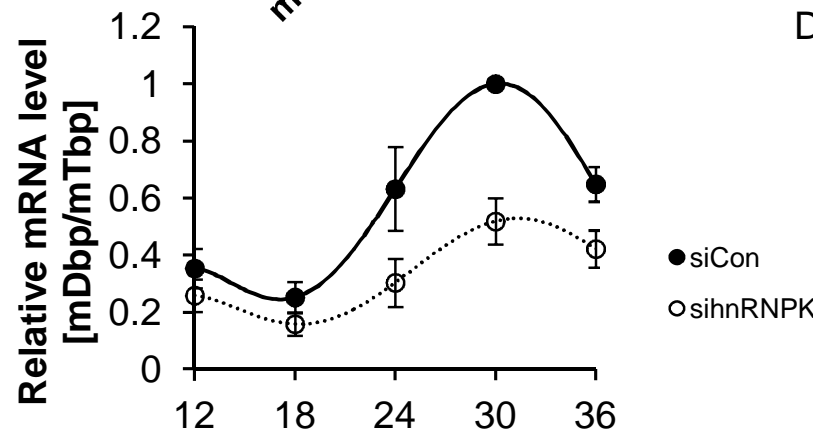

Time after Dexamethasone

synchronization [hr]

$E$

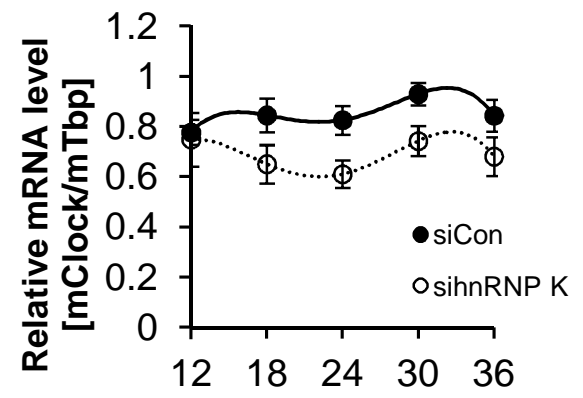

Time after Dexamethasone synchronization [hr]

$\mathrm{H}$

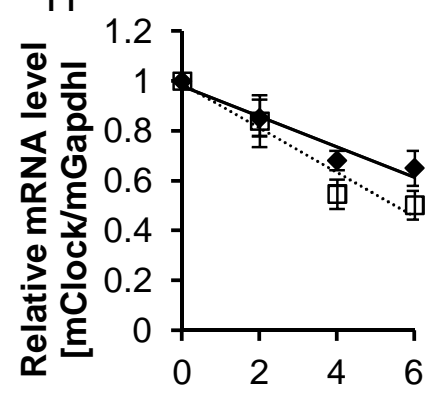

Act. D treatment [hr]

\begin{tabular}{|c|c|}
\hline & Half-life (hr) \\
\hline siCon & 16.0 \\
\hline sihnRNPK & 11.0 \\
\hline
\end{tabular}

$\mathrm{F}$

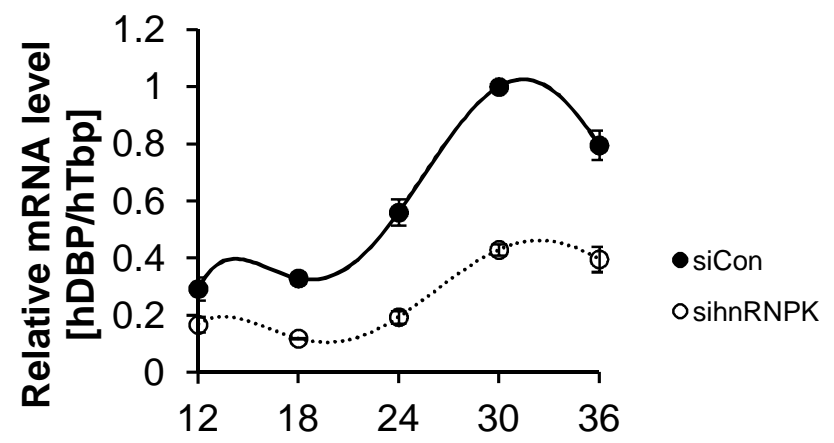

Time after Dexamethasone synchronization [hr]

G

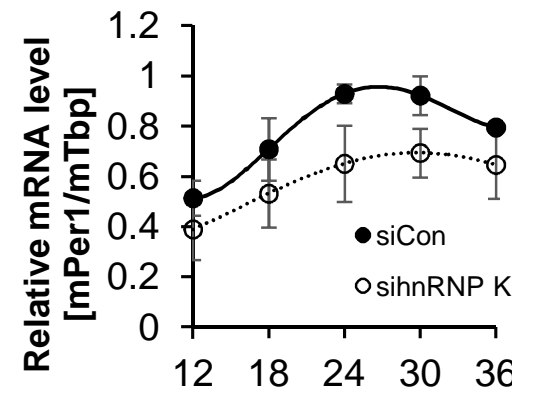

Time after Dexamethasone synchronization [hr]

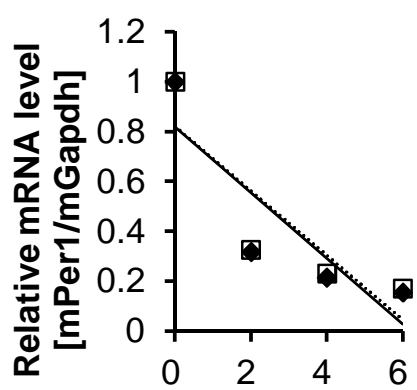

Act. D treatment [hr]

\begin{tabular}{|c|c|}
\hline & half-life $(\mathrm{hr})$ \\
\hline siCon & 2.4 \\
\hline sihnRNPK & 2.5 \\
\hline
\end{tabular}

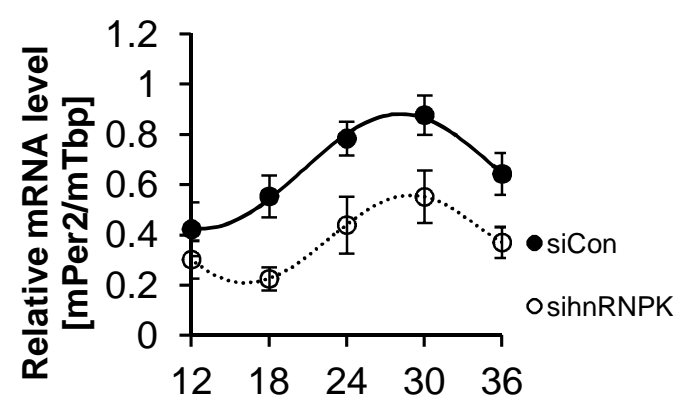

Time after Dexamethasone synchronization [hr]

$J$

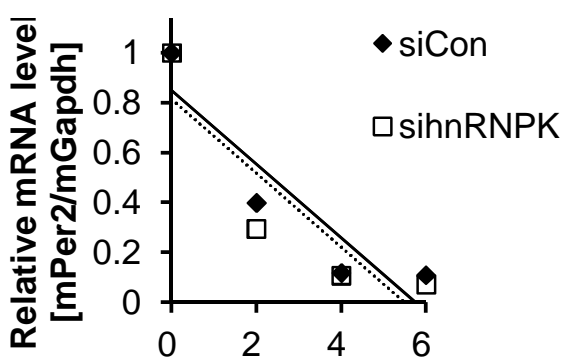

Act. D treatment [hr]

\begin{tabular}{|c|c|}
\hline & half-life $(\mathrm{hr})$ \\
\hline siCon & 2.4 \\
\hline sihnRNPK & 2.1 \\
\hline
\end{tabular}


Fig 6.

A
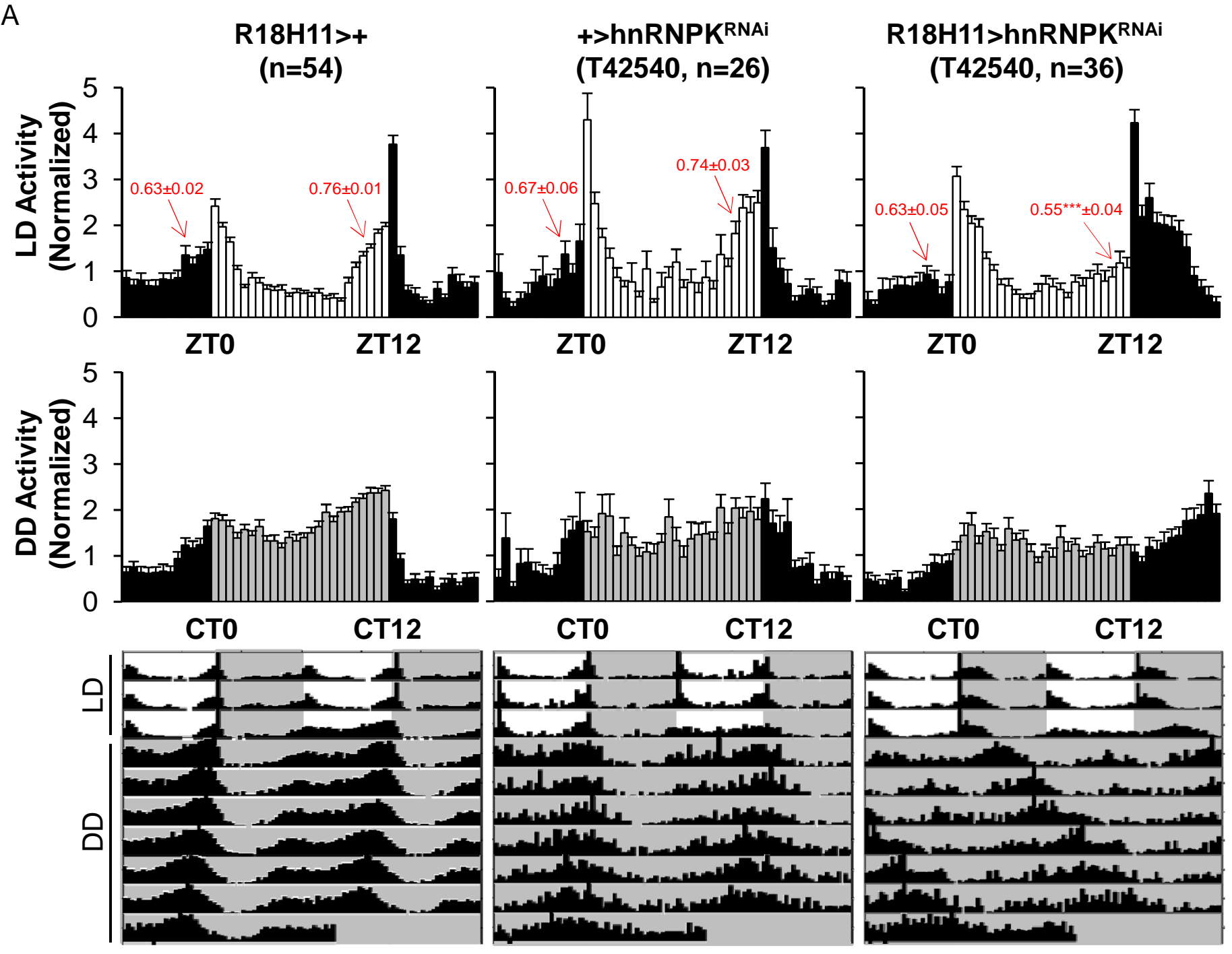

B

C
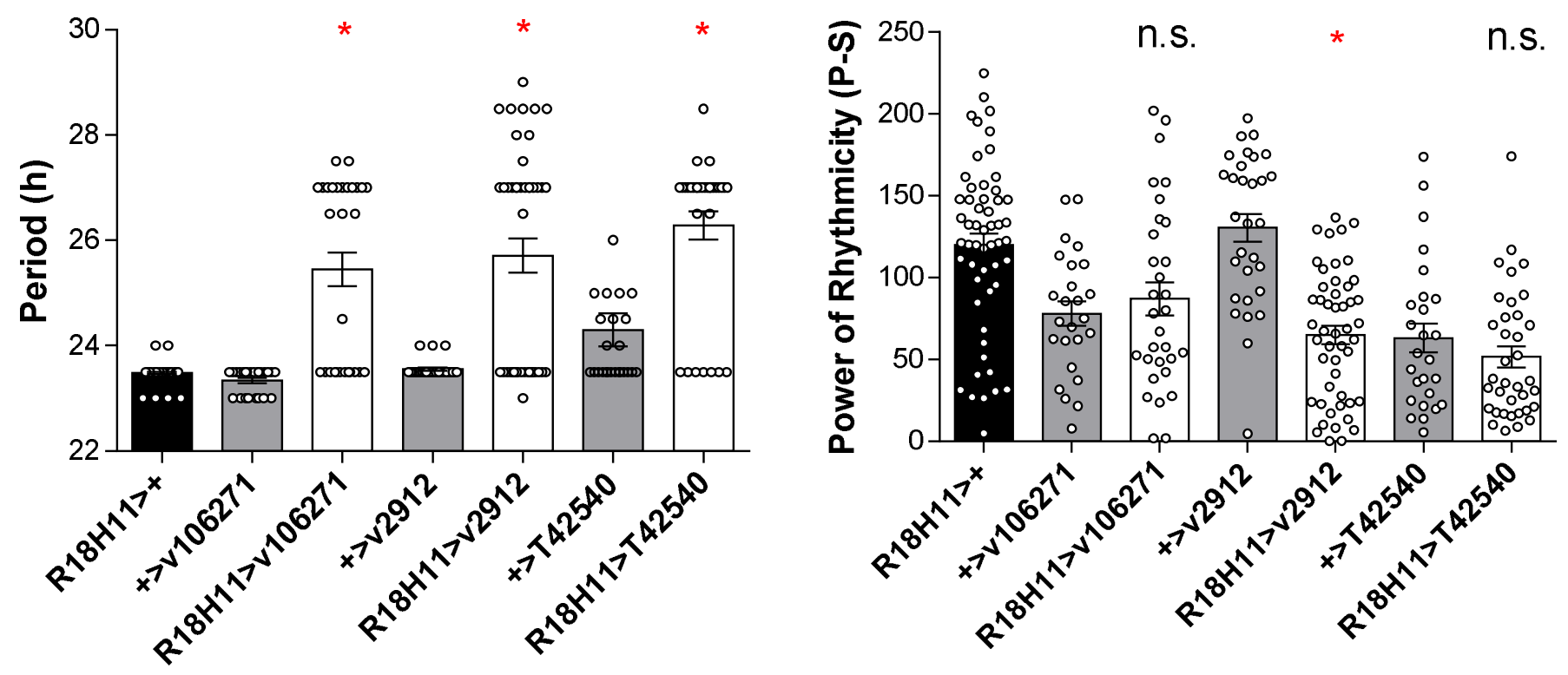